\title{
Small Sample Properties of Certain Cointegration Test Statistics: A Monte Carlo Study
}

by

Phoebus J. Dhrymes, Columbia University

Dimitrios D. Thomakos, Columbia University

November 1997

Discussion Paper Series No. 9798-08

$$
\begin{aligned}
& \text { dp } 9796-08 \\
& \text { pages: } 57
\end{aligned}
$$




\title{
SMALL SAMPLE PROPERTIES OF CERTAIN COINTEGRATION TEST STATISTICS: A MONTE CARLO STUDY
}

\author{
Phoebus J. Dhrymes \\ Dimitrios D. Thomakos \\ Columbia University
}

July 1996, This version: October 1997

\begin{abstract}
This paper reports on the results of a Monte Carlo study. The latter investigates the performance of various versions of the Conformity test ( $C C T$ ) for the existence and rank of cointegration, as given in Dhrymes (1996b), the likelihood ratio test ( $L R T$ ) as given in Johansen (J) (1988), (1991), and the stochastic trends $q_{f}(k, m)$ test (SW), as given in Stock and Watson (1988).

The design of the experiments allows for small, medium and large stationary roots, and one, two, and three unit roots. The largest system investigated is a quadrivariate $V A R(4)$.

Results based on the underlying normal theory indicate that the performance of the $C C T$ is extremely good when the null hypothesis involves the sum of, or individual, (characteristic) roots, some of which are not zero; it does not perform reliably when the sum of the roots under the null involves, in truth, all zero roots, i.e. if we are testing a null that a root, say $\lambda$ obeys $\lambda>0$ and the null is in fact true, the test performs exceedingly well. If the null, however, is in fact false it has very little power.

Results based on non-standard asymptotic theory for estimators of zero roots indicate that the $C C T$ has very good power characteristics
\end{abstract}


in detecting the rank of cointegration, but it exhibits some size distortions that can potentially lead to overestimation of the true cointegrating rank. On the other hand, both versions are robust to non normal and dependent error structures. Such results generally hold for sample sizes 100 and 500 .

For samples of size 100, the LR test performs quite well, in terms of size, when the error process is Gaussian and when small and medium stationary roots are employed in the experimental design, but does rather poorly in terms of power. The problem is magnified with large stationary roots, and/or non-normal errors. The results improve, as expected, for sample size 500 .

The SW $q_{f}(k, m)$ test performs rather poorly overall, and cannot be recommended for use in empirical applications.

Key words: Monte Carlo, Cointegration, Cointegration rank, Conformity Test, Likelihood Ratio Test.

\section{Introduction}

The purpose of this study is to investigate the small sample properties of the Conformity Cointegration Test (CCT) given in Dhrymes (1996b), the likelihood ratio test given in Johansen (1988), (1991), (J) and the Stock and Watson (SW) "filtering" test for "common trends" given in Stock and Watson (1988). A significant innovation in our work lies in the design of the Monte Carlo experiments. Using the framework provided in Dhrymes (1996), we extend the type of feasible models that may be used as Data Generating Processes (DGP). Previous studies used relatively simple specifications. This Monte Carlo study is, to the best of our knowledge, the first to employ a general $V A R$, with more than two lags, unrestricted specification on the stationary roots, and relatively mild restrictions on the parameter matrices. This will provide better information on the finite (small) sample performance of the tests under consideration, as the proposed DGP is more akin to systems encountered in empirical applications. Moreover, by comparing our results with similar results of studies that had used simpler DGP, we will assess the significance of the lag structure on various tests' performance.

A number of Monte Carlo studies have appeared in the literature including those of Yap and Reinsel (1995), ${ }^{1}$ Toda (1995) and Haug (1996). These studies explicitly study the tests we consider here but with different design approaches.

The experimental scheme of Yap and Reinsel is more closely related to ours, since in the construction of their simulated data they use a trivariate model

\footnotetext{
${ }^{1}$ They conduct a limited simulation study, as part of their work on cointegration in $\operatorname{ARM} A(p, q)$ models.
} 
and two lags. They found acceptable size properties for the LR test, but they conclude that the tests proposed by Stock and Watson are relatively weak.

The studies of Toda and Haug are done in a bivariate setting, as is the case with most previous Monte Carlo studies. The one conducted by Toda is concerned only with the LR framework. His results show unambiguously that, to achieve an acceptably good performance for the LR tests, a relatively big sample size (400) is required. He also finds that underestimation of the rank of cointegration occurs quite frequently. Moreover, he stresses the fact that the correlation structure of the model's error process plays an important role in the performance of the tests. Finally, he notes that in actual practice one typically deals with a higher dimensional system, i.e. more variables and possibly more lags; thus, the usefulness of his results as a guide to empirical practice is ambiguous. This is something our study tries to provide.

Haug's study is more extensive in the number of cointegration tests it considers, among which are both the LR tests of Johansen, and Stock and Watson's 'Common Trends' test. His design is again a bivariate one. He finds, in contrast to Yap and Reinsel, that the tests of Stock and Watson have relatively high power, and suggests that they should be considered in empirical applications. Moreover, he finds that the LR test exhibits relatively small size distortions.

Monte Carlo Studies of similar nature can also be found in Blangiewicz and Charemza (1990), who explore the empirical percentiles of cointegration tests when 'customized' testing, using empirical percentiles, is considered.

Boswijk and Frances (1992) consider, in a bivariate model, the effects on power and size of cointegration tests stemming from the dynamic specification of the model. They find that underspecification (of lags) leads to size distortions whereas overspecification leads to power loss.

Kremers, Ericsson and Dolado (1992) explore the power of the ADF (augmented Dickey-Fuller), and Engle and Granger's two step testing procedures; they operate with a bivariate design as well.

Cheung and Lai (1993) are concerned with the LR tests, again in a bivariate setting and with the use of response surface analysis. Their main consideration is the robustness of the LR tests relative to lag misspecification and non-normal errors. They find robustness in overspecification of lags. They also propose a finite sample correction for use with the asymptotic critical values. ${ }^{2}$

Haug (1993), concentrates mainly on the size distortions of residual based tests for cointegration, mainly the ADF test.

Hooker (1993) examines the trade-offs between size and power again in a simple model.

Finally, van Giersbergen (1996), considers the effects of using bootstraping

\footnotetext{
${ }^{2}$ The correction suggested is to multiply the asymptotic critical values by the factor $T /(T-$ $q n$ ) where $q$ is the dimension of the system and $n$ the number of lags.
} 
on the size properties of the LR Trace test, finding some merit in this procedure, especially in the presence of moving average components in the model.

Though the studies of Toda (1995) and Haug (1996) are concerned with models that involve a constant, and are, thus, not directly comparable to ours in terms of their general design, they are nonetheless quite useful because of their thorough experimentation. In this connection we should also note that, in their study, Yap and Reinsel (1995) assume no constant in the Data Generating Process but they include a constant in the estimation stage. This does not appear to affect significantly the performance of the tests they consider.

We organize the paper as follows: In sections 2 and 3 we present the scope of the problem under study, and the theoretical developments that motivate the $C C T$ test and its variants, for a $\operatorname{VAR}(n)$ model. We closely follow Dhrymes (1996b), but we do not present the full argumentation here; the interested reader may refer to the original reference. Section 4 explains the design of our Monte Carlo experiments. Finally, Section 5 is devoted to a discussion of the results obtained, while Section 6 provides our conclusions, along with some possible extensions of the current work.

\section{Scope of the Problem}

To set the framework of the study consider the ( $q$-element row vector) stochastic sequence $X=\left\{X_{i}: t \in \mathcal{N}_{+}\right\}$defined on the probability space $(\Omega, \mathcal{A}, \mathcal{P})$ and suppose it obeys a $V A R(n)$ specification

$$
X_{t} . \Pi(L)=\epsilon_{t}, \quad \Pi(L)=\sum_{i=0}^{n} \Pi_{i} L^{i}, \quad \Pi_{0}=I_{q}, \quad L^{0}=I,
$$

where $L$ is the usual lag operator, $I_{q}$ is the identity matrix of dimension $q$, and the unsubscripted symbol $I$ denotes the identity operator such that $X_{t} . I=X_{t} .{ }^{3}$ Dividing $\Pi(L)$ by $(L-I)$ yields

$$
\Pi(L)=\Pi(1)-(I-L) \Pi^{*}(L),
$$

where $\Pi^{*}(L)$ is a polynomial matrix operator of order $n-1$, involving functions of the matrix coefficients. If $\Pi(1)=0$, the matrix operator $\Pi(L)$ is said to have a unit root factorization, and the underlying stochastic sequence is $I(1)$ and not cointegrated. If $\Pi(1)$ is nonsingular, the underlying stochastic sequence is stationary, and thus $I(0)$. If $\Pi(1)$ is nonnull but singular, the sequence is $I(1)$ but cointegrated, and the rank of cointegration is the rank of $\Pi(1)$.

\footnotetext{
${ }^{3}$ The identity operator will be omitted when it multiplies a coefficient matrix or vector.
} 
It should also be noted that in the literature one assumes that the characteristic roots of the operator $\Pi(L)$, i.e. the roots of the determinantal equation

$$
\pi^{*}(z)=|\Pi(z)|=0,
$$

where $z$ is a complex indeterminate, obey $\left|z_{i}\right| \geq 1, i=1,2,3, \ldots, n q$. If all roots obey the strict inequality, the sequence is stationary $I(0)$; if $\Pi(L)$ has a unit root factorization,

$$
\pi^{*}(z)=(1-z)^{q}\left|\Pi^{*}(z)\right|=0 ;
$$

of course this does not automatically ensure that the determinantal component above has no additional unit roots. Finally, if the characteristic equation has precisely $r_{0}$ unit roots,

$$
\pi^{*}(z)=(1-z)^{\tau_{0}} \pi(z)=0,
$$

such that the roots of $\pi^{*}(z)=0$ obey $\left|z_{j}\right|>1, j=r_{0}+1, \ldots, n q$ or, alterntively, the roots of $|\pi(z)|=0$ (all) lie outside the unit circle. Consequently, the number of unit roots of Eq. (3) corresponds to the number of zero roots of $\Pi(1)$, and it may be noted that the remaining roots of $\Pi(1)$ are less than one in absolute value.

It follows from the preceding that to establish the existence and rank of cointegration, it is sufficient to establish the presence of zero roots for $\Pi(1)$, and their number, respectively.

The usefulness of cointegration tests for empirical researchers is dual: first, it identifies the probabilistic properties of the sequence $X$ and second, it leads to the estimation of the cointegrating matrix $B$, such that $Z=\left\{Z_{t}: t \in \mathcal{N}_{+}\right\}$ is stationary, where

$$
Z_{t}=X_{t} \cdot B
$$

In this paper we are concerned primarily with the first part above, i.e. with procedures (tests) to determine the existence and rank of cointegration, in the context of an underlying stochastic sequence, which is presumed to be $I(1)$. Testing for, and determining the rank of, cointegration involves a search for the number of unit roots in the characteristic equation of the system or, what is equivalent, the number of zero roots of $\Pi(1)$. Testing for the existence of a unit root in univariate series is commonly done using the Dickey-Fuller test, Dickey and Fuller (1979), (1981). This test was also employed in the paper by Engle and Granger (1987). Residual tests of this type were further investigated in Phillips and Ouliaris (1991), (PO) who actually examine not only the test noted above but also a number of other alternatives. PO (1988) develop tests for cointegration based on principal components. Shin (1994) 
proposes a residual based test in the context of a structural (single) equation. Stock and Watson (1988) examine a model in which the underlying stochastic sequence obeys $(I-L) X_{t}=\epsilon_{t} . C(L)$, the right member representing a "causal" moving average of infinite extent. Their objective is to determine the number of unit roots, or what they call "trends".

Testing for cointegration in the context of a VAR model has also received extensive attention in the literature. In its error correction (EC) form, Johansen (1988), (1991) pioneered in the use of maximum likelihood methods for estimating the cointegrating matrix, as well as likelihood ratio tests for establishing the presence, and rank, of cointegration. Ahn and Reinsel (1990), Johansen and Juselius (1990), Reinsel and Ahn (1992), Saikkonen (1992), Boswijk (1994), Kleibergen and van Dijk (1994), Yap and Reinsel (1995), (for ARMA(p,q) models), and Bewley and Yang (1995) all employ the VAR (EC) representation of the underlying stochastic sequence in testing for the number of unit roots.

As with the distributional theory for unit root tests in univariate series, cointegration in a multivariate context entails "non-standard" asymptotics for the limiting distributions of the test statistics, which requires a considerable degree of mathematical sophistication for its comprehension. In addition, critical values for the relevant test statistics can only be obtained via Monte Carlo approximations. ${ }^{4}$

The type of simulation experiments we undertake has been employed by other authors, but in a more circumscribed form. Previous studies had seldom employed trivariate models with more than one lag. Indeed, the most common feature of the experimental design of most Monte Carlo studies, entails a bivariate model with one lag, and occassionally a triangular structure with (a) unit $\operatorname{root}(\mathrm{s})$ and null stationary roots. By constrast, in this study we employ quadrivariate models with four lags. This is possible if we employ the structure of the design as set forth in Dhrymes (1996). The experimental design enables us to specify the roots of the characteristic equation of the system arbitrarily and, subsequently, determine the elements of the matrices $\Pi_{i}$ of Eq. (1). This is not the most general possible design, in that the matrices in question need to obey certain triangularity conditions. Still, the experimental design we employ enables us to assess the relevance of the size of the stationary roots in determining the small sample performance of the tests examined. Moreover, by comparing our results with similar results in previous studies we may be able to determine whether the dimension of the system, and/or the number of lags specified, has any bearing on the small sample performance of various

\footnotetext{
${ }^{4} \mathrm{~A}$ by-product of this study, is a new set of critical values for both the Conformity Test and the Likelihood Ratio Test. They appear in Dhrymes (1998), (Appendix II to Ch. 6). They are based on 2000 observations and 20,000 replications and are, thus, more accurate than anything that has hitherto appeared in the literature.
} 
cointegration tests.

\section{Theoretical Framework}

Consider again the stochastic sequence $X$ defined in Eq. (1), which we repeat for clarity,

$$
X_{t} \Pi(L)=\epsilon_{t}, \quad t=1,2,3, \ldots \ldots,
$$

where $\epsilon=\left\{\epsilon_{t}: t \in \mathcal{N}_{+}\right\}$is a $q$-element (multivariate) white noise process with mean zero and positive definite covariance matrix $\Sigma$. In view of Eq. (2) we may write the model in Eq. (1) in its Error Correction form

$$
\Delta X_{t} .=-X_{t-1} \Pi(1)+\sum_{i=1}^{n-1} \Delta X_{t-i} \cdot \Pi_{i}^{*}+\epsilon_{t} .
$$

Using the notation

$$
P=\left(X_{t}\right), \quad X=\left(\Delta X_{t-1 .}, \Delta X_{t-2 \cdot}, \cdots, \Delta X_{t-n+1}\right), J=\left(-\Pi(1)^{\prime}, \Pi^{*^{\prime}}\right)^{\prime}
$$

where $\Pi^{*}=\left(\Pi_{0}^{*^{\prime}}, \ldots, \Pi_{n-1}^{*^{\prime}}\right)^{\prime}$, we may write the entire sample on the EC form of Eq. (6) as

$$
\Delta P=-P_{-1} \Pi(1)+X \Pi^{*}+U, \quad U=\left(\epsilon_{t}\right) .
$$

Under the cointegration hypothesis, the matrix $\Pi(1)$ is of reduced rank $r=$ $q-r_{0}$, where $r_{0}$ is the number of unit roots of the characteristic equation of the system. In the likelihood ratio context of Johansen (1988), (1991), one uses the rank factorization theorem to write

$$
\Pi(1)=B \Gamma^{\prime}
$$

where $B, \Gamma$ are both $q \times r$ matrices of rank $r$. In that context, one further assumes that the $\epsilon$-process is Gaussian, maximizes the likelihood function, thus estimating the matrix of cointegrating vectors $B$, and the matrix $\Gamma$ of the so called 'factor loadings'. The maximized value of the likelihood function is

$$
L\left(B, \Gamma, \Pi^{*}, \Sigma\right)=c_{0}-\frac{T}{2} \sum_{i=1}^{r} \ln \left(1-\hat{\lambda}_{i}\right),
$$

where the $\hat{\lambda}_{i}$ are the $r$ largest roots of

$$
\begin{aligned}
0 & =\left|\lambda V^{\prime} V-V^{\prime} W\left(W^{\prime} W\right)^{-1} W^{\prime} V\right|, \quad V=N P_{-1}, \quad W=N \Delta P, \\
N & =I_{T}-X\left(X^{\prime} X\right)^{-1} X^{\prime} .
\end{aligned}
$$


The test for cointegration of rank $r$ is then a likelihood ratio test (LRT) that the remaining $r_{0}=q-r$ roots are null.

The conformity cointegration test $(C C T)$, on the other hand, operates with the unrestricted estimator of $\Pi(1)$, which is given by

$$
\hat{\Pi}(1)=-\left(V^{\prime} V\right)^{-1} V^{\prime} W=\Pi(1)-\left(V^{\prime} V\right)^{-1} V^{\prime} U,
$$

does not require that the $\epsilon$-process be Gaussian, and relies on the fact that $T^{\alpha}[\hat{\Pi}(1)-\Pi(1)] \stackrel{P}{\rightarrow} 0$, for any $\alpha<1 .{ }^{5}$ The CCT is, thus, a semiparametric test in that it relies only on the basic definition of cointegration, i.e. on the fact that if the sequence is cointegrated, the entity $z_{t}=X_{t} . \mathrm{II}(1)$ is stationary and, consequently, $(1 / T) \Pi(1)^{\prime} P_{-1}^{\prime} P_{-1} \Pi(1)$ converges to a $q \times q$ singular matrix, of rank equal to the rank of cointegration. It is shown in Dhrymes (1996b) that if we define

$$
\hat{M}=\frac{1}{T} \hat{\Pi}(1)^{\prime} P_{-1}^{\prime} P_{-1} \hat{\Pi}(1)
$$

and if $M(0)$ is the probability limit of $\hat{M}$,

$$
\frac{1}{\sqrt{T}} \operatorname{vec}[\hat{M}-M(0)]=\frac{1}{\sqrt{T}} \sum_{t=1}^{T} g_{t} \stackrel{\mathrm{d}}{\rightarrow} g \sim N\left(0, \Psi^{*}\right)
$$

where

$$
g_{t}=\left[\hat{z}_{t-1}^{\prime} \otimes \hat{z}_{t-1 \cdot}^{\prime}-m(0)\right], \quad m(0)=\operatorname{vec}[M(0)], \quad \Psi^{*}=\lim _{T \rightarrow \infty} E\left(g_{t} g_{t}^{\prime}\right) .
$$

Note that the covariance matrix $\Psi^{*}$ is singular since it contains redundant elements, such as e.g. the variance and covariances of the elements $\hat{m}_{i j}$ and $\hat{m}_{j i}$, for $i \neq j$. This, however, is of little consequence since we may remove the redundant elements quite easily. To this end, note that if $S$ is a $q(q+1) / 2 \times q^{2}$ selection matrix that extracts the distinct elements of $\operatorname{vec}(\hat{M})$, the relevant covariance matrix is obtained as

$$
\Psi=S \Psi^{*} S^{\prime}
$$

It is also shown in Dhrymes (1996b) that the (ordered) characteristic roots of $\hat{M}$, contained in the diagonal matrix $\tilde{\Lambda}$, obey

$$
\sqrt{T} d^{*}[\tilde{\Lambda}-\Lambda] \sim d^{*}\left[\sqrt{T} Q^{\prime}[\hat{M}-M(0)] Q\right],
$$

where the notation $d^{*}[A]$ indicates the diagonal elements of the matrix $A$, and $Q$ is the orthogonal matrix of the decomposition $M(0)=Q \Lambda Q^{\prime}$.

\footnotetext{
${ }^{5}$ Occassionally this property is referred to in the literature as "superconsistency".
} 
We are now in a position to formulate the first variant of the $C C T$. Denote by $\hat{\lambda}_{i}, i=1,2, \ldots, q$, the roots of $\hat{M}$ arranged in decreasing order of magnitude and note that, under cointegration, at least $\hat{\lambda}_{1}$ converges to a strictly positive limit, so that $\operatorname{tr} M(0)>0$. Let $S_{1}$ be another selection $\left(q \times q^{2}\right)$ matrix that selects from $\operatorname{vec}(\hat{M})$ its diagonal elements. The marginal limiting distribution of the (centered) diagonal elements is evidently normal with mean zero and covariance matrix

$$
\Psi_{1}=S_{1} \Psi^{*} S_{1}^{\prime} .
$$

We are now in a position to formulate a general standard test for the presence of cointegration, based on the statistic

$$
C C T(q)=\frac{T^{1 / 2}(\operatorname{tr}[\hat{M}-M(0)])}{\sqrt{e^{\prime} \Psi_{1} e}} \stackrel{\mathrm{d}}{\rightarrow} N(0,1),
$$

where $e$ is a $q$-element column vector of unities.

Remark 1. In applications, the value of $\operatorname{tr} M(0)$ is, of course, unknown and the question arises as to precisely how the test is to be carried out. The natural null hypothesis is $\operatorname{tr} M(0)=0$; however, this is the hypothesis that all the roots of $\Pi(1)$ are null so that for all intents and purposes $\Pi(1)=0$. The preceding discussion indicates that this condition implies that the underlying sequence is $I(1)$, and not cointegrated. Hence, the distribution theory under which we operate is invalid. Thus, what we need is to formulate the test in terms of the null hypothesis

$$
H_{0}: \operatorname{tr} M(0)>0 \text {, }
$$

as against the alternative

$$
H_{1}: \operatorname{tr} M(0)=0 \text {. }
$$

If the hypothesis is rejected we take it to mean that the sequence is $I(1)$, and not cointegrated. If it is accepted we take the result to mean that the underlying sequence is cointegrated. Using the limiting distribution theory alluded to above, let $\operatorname{tr} \hat{M}$ be the test statistic and note that, under the null we have, asymptotically,

$$
\mathcal{P}\left(A^{*}\right)=1-\alpha, \text { where } A^{*}=\left\{\omega: T^{1 / 2}\left|\frac{\operatorname{tr} \hat{M}-\operatorname{tr} M(0)}{s}\right| \leq 1.96\right\},
$$

where $T$ is the sample size and $s$ is the estimator of the standard deviation as given by the limiting distribution. The relationship of Eq. (20) implies that (for large $T$ ) the random interval

$$
\left[\operatorname{tr} \hat{M}-1.96 \frac{s}{\sqrt{T}}, \operatorname{tr} \hat{M}+1.96 \frac{s}{\sqrt{T}}\right]
$$


covers the true parameter with probability, approximately, $1-\alpha$. Taking this interval, then, as the acceptance region of the test we have the following procedure: If with a given sample the interval above does not contain zero, we accept the null; if it does we reject. This then is a test of (approximate) size $\alpha$.

Alternatively, we may use a device similar to the employed by Phillips and Ouliaris (1988) in connection with their principal components based test for cointegration. In our case this would involve formulating the hypothesis as

$H_{0}: \operatorname{tr} M(0)=\gamma, \gamma$ arbitrarily small,

as against the alternative

$H_{1}: \operatorname{tr} M(0) \neq \gamma$

where $\gamma$ is an arbitrarily small number such as .01 or .001 . The reason this arbitrariness does not materially affect the results is that in this case, as well as in Phillips and Ouliaris, the test in question is consistent.

From a practical point of view, it makes no particular difference whether we choose $\gamma$ to be zero or .01 or .001 , since in the presence of cointegration tr $\hat{M}$ overwhelms $\gamma$. The reason for its insertion, however, is simply to uphold the applicability of the limiting distribution to the null. Notice that if we state the null as $\operatorname{tr} M(0)=0$, this form of the null is not an admissible hypothesis, given the conditions employed in deriving the limiting distribution. On the other hand $\operatorname{tr} M(0)=\gamma$ is, strictly speaking, admissible. Consequently, this device preserves the formal niceties, and at the same time gives us a particularly simple procedure in testing for the presence of cointegration.

If one is uncomfortable with this last formulation one may always use the confidence interval interpretation noted earlier.

To test whether a particular root of $M$ is null, one needs the limiting distribution of the characteristic roots, which may be found in Dhrymes (1996b). In connection with that derivation we note that the nonnull characteristic roots, say $\hat{\lambda}=\left(\hat{\lambda}_{1}, \hat{\lambda}_{2}, \hat{\lambda}_{3}, \ldots, \hat{\lambda}_{r}\right)^{\prime}$ have the limiting distribution

$$
\sqrt{T}(\hat{\lambda}-\lambda) \rightarrow N\left(0, \Sigma_{\hat{\lambda}}\right)
$$

while the null roots, as well as their limiting covariance matrix, both converge to zero in the following sense. Let $\lambda_{*}$ consist of the $r_{0}$ remaining roots of $M(0)$. If we focus our attention on $\hat{M}^{*}=(1 / T) \Pi(1)^{\prime} P_{-1}^{\prime} P_{-1} \Pi(1)$, which has the same limiting behavior as $\hat{M}$, the entire preceding limiting distribution argument remains valid. Then

$$
\sqrt{T}\left(\hat{\lambda}_{*}-\lambda_{*}\right) \stackrel{\mathrm{d}}{\rightarrow} N\left(0, \Sigma_{\hat{\lambda}_{*}}\right) .
$$


It is shown in Dhrymes (1996b) that as $\lambda_{*} \rightarrow 0, \Sigma_{\hat{\lambda}_{*}} \rightarrow 0$, and $\sqrt{T} \hat{\lambda}_{*} \rightarrow 0$, at least in probability. Thus, in terms of the framework provided above, no formal test is possible for the null roots. Consequently, we need to examine the question of how we should normalize their estimators in order to establish a nontrivial limiting distribution.

In view of the preceding discussion a test of the hypothesis that the rank of cointegration is at least $r$, may be carried out through the test statistic

$$
C R T\left(r_{0}+1\right)=\frac{\sqrt{T}\left(\sum_{j=r}^{q} \hat{\lambda}_{j}-\gamma\right)}{s_{q-r+1}}
$$

where $s_{q-r+1}$ is the estimated standard deviation of the sum in the numerator. If in fact $\lambda_{r}>0$, and the test results in rejection, we shall conclude that at least $\lambda_{r}>0$, so that there are at most $q-r=r_{0}$ zero roots and, consequently, that the rank of cointegration is at least $r$. Thus, the test statistic CRT(i) can be used, sequentially, in order to test for the rank of cointegration. The procedure will fail to produce meaningful results when all the summands are in fact null, i.e. when in truth $\sum_{j=r}^{q} \lambda_{j}=0$.

\subsection{Limiting Distribution of Zero Root Estimators}

To tackle this problem it turns out that it is more convenient to operate with the characteristic roots of $\hat{M}$ in the metric of $\Sigma$, the covariance matrix of the structural errors. Thus, we consider the roots of

$$
|\lambda \Sigma-\hat{M}|=0 .
$$

It is shown in Dhrymes (1996b), (1998) that the estimator of the non zero roots remains as before, mutatis mutandis, while the limiting distribution of the estimator of zero roots is nonstandard and, moreover, the following is true:

i. A test of the hypothesis that the rank of cointegration is $r$, as against the alternative that it is greater than $r$, i.e. a test of the null hypothesis that there are $r_{0}=q-r$ zero roots, as against the alternative that there less that $r_{0}$, may be based on

$$
T \sum_{i=r+1}^{q} \hat{\lambda}_{i} \stackrel{\mathrm{d}}{\rightarrow} \operatorname{tr} \Upsilon^{\prime} K^{-1} \Upsilon, \quad K=\int_{0}^{1} B(r)^{\prime} B(r) d r, \quad \Upsilon=\int_{0}^{1} B(r)^{\prime} d B_{1}(r),
$$

where $B, B_{1}$ are SMBM (standard multivariate Brownian motion), represented as row vectors with $q$ and $r_{0}$ elements, respectively. 
ii. A test that the rank of cointegration is $r$ as against the alternative that it is $r+1$, i.e. a test that there are $r_{0}$ zero roots as against the alternative that there are at most $r_{0}-1$ zero roots, may be carried out through the statistic $T \hat{\lambda}_{r}$.

Remark 2. Notice that the limiting distribution of the test statistic in i above is simply the distribution of the sum of the roots of the system

$$
\left|\rho I_{\tau_{0}}-\Upsilon^{\prime} K^{-1} \Upsilon\right|=0,
$$

while the test statistic in ii is based on the largest root of that system. For this reason the first test is said to be the trace test, while the second is said to be the maximal root test for cointegration rank. In the Monte Carlo results, the test statistic for the trace test is denoted by $C R T_{Z}\left(r_{0}\right)$, while the test statistic for the maximal root based test is denoted by $M R C_{Z}\left(r_{0}\right)$.

Notice, further, that this distribution is not the same as that given in Johansen and Juselius (1990), in Osterwald-Lenum (1992), or in Saikkonen (1992), although it is certainly of the same genre. In Johansen, the equivalent of $B$ and $B_{1}$ are of the same dimension, while in the conformity context the first is of dimension $q$ and the second of dimension $r_{0}$. Thus, in the Johansen context the test statistic for a unit root in a two equation system has precisely the same distribution as a test statistic for a unit root in a $q$-equation system. Or, put in slightly different terms, a test statistic for three unit roots in a four-equation system has the same distribution as the test statistic for three unit roots in a ten-equation system. Thus, in empirical applications it is more likely that in larger systems we would find a higher incidence of cointegration than is perhaps inherent in the data. The distribution of the conformity test statistic, however, has two parameters, the dimension of the system, $q$, and the number of unit roots, $r_{0}$.

Finally, before we deal with the Monte Carlo design and results, we present a brief explanation of how the characteristic roots in $C C T$ are related to the characteristic roots in the likelihood ratio tests given in Johansen (1988), (1991).

Remark 3. If, as in Johansen (1988), (1991), we concentrate the likelihood function, we ultimately find that we need to minimize with respect to $B$, which is a $q \times r$ matrix of rank $r$, the determinant

$$
D(B)=\left|W^{\prime} W-W^{\prime} V B\left(B^{\prime} V^{\prime} V B\right)^{-1} B^{\prime} V^{\prime} W\right| .
$$

After considerable manipulation, we determine that this requires us to obtain the (characteristic) roots and vectors of

$$
\left|\lambda V^{\prime} V-V^{\prime} W\left(W^{\prime} W\right)^{-1} W^{\prime} V\right|=0 .
$$


The solution to the problem is to select the $r$ largest roots, and their associated characteristic vectors. The latter serve as the estimator of the matrix $B$ in the rank factorization $\Pi(1)=B \Gamma^{\prime}$. Note further that, under the null of cointegration, the remaining roots are zero. Hence, in the LR procedure the rank of cointegration is simply the number of positive roots in the limit version of Eq. (26). It may be shown that this procedure is equivalent to defining the matrix

$$
\hat{M}_{1}=\frac{1}{T} \hat{\Pi}(1)^{\prime} V^{\prime} V \hat{\Pi}(1)
$$

and obtaining the (nonnull) roots of $\hat{M}_{1}$ in the metric of $W^{\prime} W / T$, i.e. the roots of

$$
\left|\lambda W^{\prime} W-W^{\prime} V\left(V^{\prime} V\right)^{-1} V^{\prime} W\right|=\left|\lambda W^{\prime} W-T \hat{M}_{1}\right|=0 .
$$

In the conformity test context, the rank of cointegration is determined by the positive roots in the limit version of

$$
|\lambda I-T \hat{M}|=0, \text { or }|\lambda \Sigma-T \hat{M}|=0 .
$$

Thus, basically, the LR (Johansen) procedure determines the rank of cointegration by the number of positive roots of the limit of $\hat{M}_{1}$, in the metric of $M_{x_{0} x_{0}}^{*}$. The latter is simply the (a.c.) limit of $W^{\prime} W / T$, and thus the conditional covariance matrix of $\Delta X_{t}$, conditioned on the $\sigma$-algebra generated by the lagged differences. It is this feature that renders such roots less than unity, and thus impedes the effective separation of roots in empirical applications. By contrast, the conformity approach determines the rank of cointegration by the positive characteristic roots of the limit of $\hat{M}$, which is the unconditional covariance matrix of the cointegral vector, $z_{t}=X_{t} \Pi(1)$, in the metric of the identity matrix, or the metric of the structural error matrix. Thus, the roots are not compressed by measuring them in terms of "units" of a possibly large covariance matrix, and this contributes to a very effective separation of the zero roots in an empirical context.

\section{Monte Carlo Results}

\subsection{Preliminaries}

In this section we present results from a simulation study of the finite sample behavior of the various tests for cointegration discussed in earlier sections. The general model we consider is that given by Eq. (1). We use two Data Generating Processes (DGP), a trivariate $V A R(3)$, and a quadrivariate $V A R(4)$. To our knowledge such specifications have not been employed in previous Monte Carlo 
studies and, in particular, no previous study has employed as many lags as we have incorporated in our design.

The precise method of construction of our DGP may be found in Dhrymes (1996a). The DGP always contain $r_{0}<q$ unit roots, so we have built into our design cointegration of rank $r=q-r_{0}$.

Two sample sizes are considered throughout, $T=100$, and $T=500$. The choice of sample sizes is meant to provide information on the performance of the tests examined above in the setting frequently used in empirical work, $(T=100)$, and to gauge the performance of such tests in samples large enough so that the limiting distribution results may be presumed to hold reasonably accurately, $(T=500)$. The number of replications $(\mathrm{R})$ is set to 3,500 for the first sample size, and 1,500 for the second one; the limited number of replications in that case is simply due to excessive computional costs. All computations were done using the GAUSS programming language.

In most previous Monte Carlo studies, the DGP are constrained either to be simple $V A R(1)$ or $V A R(2)$ processes with "full" coefficient matrices or to be special formulations that involve restrictions on the system's parameters; a popular parametrization is the one initially used by Banerjee et al. (1986), and subsequently by many other authors. Another variant occsionally employed is the so called "triangular" system representation, initially proposed by Phillips (1991) for studying the asymptotic properties of a cointegrated ECM. The major differences of the DGP we employ, in comparison to previous formulations, are the explicit inclusion of more lags, thus of more complicated transient dynamics, and the specification at will of (the inverse) of all the roots of $|\Pi(z)|=0$. In this fashion we specify explicitly and precisely the salient features of the "forces" that drive the dynamics of the system, $(V A R(n))$, and which may well play an important role in determining the small sample behavior of various test statistics. Moreover, the ability to operate in this direct fashion allows us more flexibility in specifying different stationary root configurations.

\subsection{Experimental Design}

As mentioned above, an important aspect of the experimental design is the choice of the stationary roots in the determinantal equation $|\Pi(z)|=0$. In most previous Monte Carlo studies a $V A R(1)$ specification is employed. In that context, it is rather trivial to choose coefficient matrices, $\Pi_{i}$, such that Eq. (3) has a certain number of unit roots, and stationary roots of any desired magnitude. However, for higher order systems more care must be exercised. If we want to obtain the coefficient matrices corresponding to some set of prespecified roots, the problem is anything but trivial. This problem is considered in Dhrymes (1996a), whose solution we have employed in constructing a variety 
of parametric configurations (PC).

We have worked with a trivariate $V A R(3)$, and a quadrivariate $V A R(4)$. For the baseline experiments we have chosen error processes which are i.i.d. normal with mean zero and covariance matrix

$$
\Sigma_{3}=\left[\begin{array}{ccc}
1.0 & .50 & .75 \\
.50 & 1.0 & .85 \\
.75 & .85 & 1
\end{array}\right], \quad \Sigma_{4}=\left[\begin{array}{llll}
1.0 & .50 & .65 & .75 \\
.50 & 1.0 & .55 & .85 \\
.65 & .55 & 1.0 & .45 \\
.75 & .85 & .45 & 1.0
\end{array}\right]
$$

for the trivariate and quadrivariate specifications, respectively. In addition to the baseline error structure, we have considered errors which are i.i.d. centered chi-square, and zero mean errors which are $M A(4)$.

The $\mathrm{PC}$ for these specifications are given below in Tables 1, (trivariate $V A R(3)$ ), and 2, (quadrivariate $V A R(4)$ ). The parametric configuations exhibited therein consist of specifications with one, two, and three unit roots, each combined with low, moderate, and large stationary roots.

Table 1

TRIVARIATE $V A R(3)$

Inverse of the Roots of $|\Pi(z)|=0$, and Characteristic Roots of true $M$.

\begin{tabular}{||r|r|r|r|r|r||}
\hline \hline PC1 & PC2 & PC3 & PC4 & PC5 & PC6 \\
\hline \hline \multicolumn{6}{|c||}{ Roots in Descending Order } \\
\hline \hline 1.00 & 1.00 & 1.00 & 1.00 & 1.00 & 1.00 \\
\hline 0.43 & 1.00 & 0.82 & 1.00 & 0.95 & 1.00 \\
\hline 0.39 & 0.43 & 0.79 & 0.81 & 0.79 & 0.95 \\
\hline 0.32 & 0.35 & 0.68 & 0.72 & 0.68 & 0.66 \\
\hline 0.29 & 0.31 & 0.61 & 0.63 & 0.55 & 0.58 \\
\hline 0.22 & 0.26 & 0.55 & 0.56 & 0.47 & 0.49 \\
\hline 0.20 & 0.22 & 0.49 & 0.49 & 0.35 & 0.34 \\
\hline 0.15 & 0.12 & 0.43 & 0.43 & 0.20 & 0.25 \\
\hline 0.10 & 0.10 & 0.40 & 0.40 & 0.15 & 0.15 \\
\hline \hline \multicolumn{7}{|c|}{ ROOTS OF } & TRUE & M \\
\hline \hline 2.4695 & 11.2453 & 1.7523 & 0.07452 & 5.5457 & 0.1617 \\
\hline 0.1585 & 00.0000 & 0.1304 & 0.00000 & 0.0211 & 0.0000 \\
\hline 0.0000 & 00.0000 & 0.0000 & 0.00000 & 0.0000 & 0.0000 \\
\hline \hline
\end{tabular}


In the trivariate design, $\mathrm{PC} 1, \mathrm{PC} 3$, and $\mathrm{PC} 5$ have one unit root and small, medium, and large stationary roots, respectively; PC2, PC4, and PC6 have two unit roots, and small, medium, and large stationary roots respectively. It should be noted that PC4 has two unit roots, but the (only) nonzero root of the true $M$ is .07; this implies that this particular PC design represents a "nearly" noncointegrated $I(1)$ process, although arguably one could claim that this is onlya matter of "scaling". In line with this interpretation, it is interesting to note that for $T=100$, the $C C T$ framework gives comparable root ratios between the pairs, first/ second, and second/third (14 vs. 11).

Table 2

QUADRIVARIATE $V A R(4)$

Inverse of the Roots of $|\Pi(z)|=0$, and Characteristic Roots of true $M$.

\begin{tabular}{|c|c|c|c|c|c|c|c|}
\hline $\mathrm{PC} 1$ & $\mathrm{PC} 2$ & PC3 & $\overline{\mathrm{PC} 4}$ & $\mathrm{PC5}$ & $\begin{array}{l}\text { PC6 } \\
\end{array}$ & $\mathrm{PC} 7$ & PC8 \\
\hline \multicolumn{8}{|c|}{ Roots in Descending Order } \\
\hline 1.00 & 1.00 & 1.00 & 1.00 & 1.00 & $\overline{1.00}$ & 1.00 & 1.00 \\
\hline 0.48 & 1.00 & 1.00 & 0.95 & 1.00 & 0.80 & 1.00 & 1.00 \\
\hline 0.45 & 0.48 & 1.00 & 0.85 & 0.95 & 0.77 & 0.80 & 1.00 \\
\hline 0.41 & 0.45 & 0.48 & 0.77 & 0.85 & 0.75 & 0.77 & 0.80 \\
\hline 0.39 & 0.41 & 0.45 & 0.74 & 0.77 & 0.71 & 0.75 & 0.77 \\
\hline 0.33 & 0.39 & 0.41 & 0.67 & 0.74 & 0.66 & 0.71 & 0.75 \\
\hline 0.27 & 0.33 & 0.39 & 0.48 & 0.67 & $\overline{0.62}$ & 0.66 & 0.71 \\
\hline 0.21 & 0.27 & $\overline{33}$ & 0.37 & 0.48 & 0.56 & $\overline{0.62}$ & 0.66 \\
\hline 0.17 & 0.21 & 27 & 0.33 & 0.37 & 0.51 & 0.56 & 0.62 \\
\hline 0.13 & 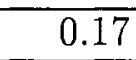 & 21 & 0.27 & 0.33 & 0.44 & 0.51 & 0.56 \\
\hline 0.10 & 0.13 & 0.17 & 0.25 & 0.27 & 0.41 & 0.44 & 0.51 \\
\hline-0.24 & 0.24 & 0.42 & 0.42 & -0.47 & -0.47 & -0.47 & -0.47 \\
\hline-0.30 & -0.30 & -0.30 & -0.55 & -0.55 & -0.53 & -0.53 & -0.53 \\
\hline-0.36 & -0.36 & -0.36 & -0.72 & -0.72 & -0.60 & -0.60 & -0.60 \\
\hline-0.44 & -0.44 & -0.44 & -0.81 & -0.81 & -0.68 & -0.68 & -0.68 \\
\hline-0.46 & -0.46 & -0.46 & -0.90 & $-0 . \overline{90}$ & -0.74 & $\overline{-0.74}$ & -0.74 \\
\hline \multicolumn{8}{|c|}{ ROOTS OF TRUE $\mathrm{M}$} \\
\hline$\overline{18.4054}$ & 8.4657 & 2.4648 & $\overline{58.2046}$ & 2.0882 & 9.9347 & $\overline{24.0416}$ & 21.0220 \\
\hline 0.5839 & 0.2106 & 0.0000 & 0.3289 & 0.0340 & 0.2531 & 0.0289 & 0.0000 \\
\hline 0.1300 & 0.0000 & 0.0000 & 0.0159 & 0.0000 & 0.0454 & 0.0000 & 0.0000 \\
\hline 0.0000 & 0.0000 & 0.0000 & 0.0000 & 0.0000 & 0.0000 & 0.0000 & 0.0000 \\
\hline
\end{tabular}


By contrast, in the LRT framework the comparable numbers are 3 and 7 . Similarly, in PC6 we also have two unit roots, but the largest root of the true $M$ is only .16, which implies that this design represents a cointegrated, but a "slow" developing, $I(1)$ process.

Basically, the same arrangement was followed in the quadrivariate design, except that it proved exceedingly difficult to obtain a parametric configuration with three unit roots and large stationary roots, i.e. roots of about $|.95|$.

Because we wished to examine the bias of the estimated roots of $M$, we have also provided in the tables the roots of the true $M$. An interesting feature of both specifications is the wide variabilty in the largest root of the true $M$.

The likelihood ratio (LR) (Johansen's), and Stock and Watson's (SW) tests are constructed as they are described in the respective references. We note that in obtaining the SW $q_{f}(k, m)$ test we have used, for filtering, a lag polynomial of degree two for the trivariate $V A R(3)$, and degree three for the quadrivariate $V A R(4)$. This choice of lags is simply based on the number of lags in the specification of the experimental design. Critical values for these tests were obtained, respectively, from our own tabulations, Dhrymes (1998), Appendix II of Chapter 6, and Table 1 of Stock and Watson (1988).

In all of our experiments we estimate the "true" model, i.e. we always use the same number of lags as the DGP in question, and no constant. We did not use any method of selecting the lag length, as it is commonly done in other studies, because we did not wish to investigate the properties of a misspecified model. Neither did we include a constant, since in practical applications we can always work with series that have been detrended or demeaned. For the $A R M A(4,4)$ specification we have used the error process

$$
u_{t .}=\epsilon_{t} \cdot A(L), \quad A(L)=\sum_{j=0}^{4} A_{j} L^{j} .
$$

The polynomial operator was invertible, the inverse of the roots of the characteristic equation $|A(z)|=0$ was bounded by .8 , and the $\epsilon$-process was one of i.i.d. normal vectors with mean zero and covariance matrix as given in Eq. (28).

\section{Estimation of the Roots of M: Bias Assessment}

\subsection{Normal Errors (i.i.d.)}

As we mentioned in section 2, a feature of the $M$ matrix is that the number of its zero characteristic roots corresponds to the number of unit roots in the system, 
i.e. in $|\Pi(z)|=0$. Thus, we expect to see a clear indication of the existence of cointegration just by visual inspection of the characteristic roots of M. Since these roots correspond to an 'unconditional' covariance matrix, their magnitude is not restricted. This is in contrast to J's procedure, where the characteristic roots of the appropriate matrix correspond to the roots of a conditional in the metric of an unconditional covariance matrix and are, thus, constrained to be less than one. As, at this stage, we do not have the 'true' characteristic roots corresponding to J's procedure we will have to rely on estimated quantities.

We begin the presentation of our results with the Monte Carlo means of J's roots and the means of the estimated roots of $\mathrm{M}$. Results in this subsection are for the case where the DGP has i.i.d. normal errors. Tables 3 and 4 contain the results for the trivariate $\operatorname{VAR}(3)$ and Tables 5 and 6 those for the quadrivariate $\operatorname{VAR}(4)$ model.

In Tables $3 \mathrm{~A}$ and $3 \mathrm{~B}$, below, we have the Monte Carlo means of the estimated roots of $\mathrm{M}$ for the trivariate $\operatorname{VAR}(3), \mathrm{T}=100$, and $\mathrm{T}=500$, respectively. There is always positive bias in the estimation of roots for all PC. The bias of estimators corresponding to the zero roots of the true $\mathrm{M}$ is generally of the order of $10^{-2}$. Exception is the estimator of the smallest root when the sample is 500 .

The use of the metric of $\Sigma$ enhances the separation of the zero from the non-zero roots in $\mathrm{PC} 1$ and $\mathrm{PC} 2$, which both have small stationary roots, but has almost no effect for the other PC. For example, for $\mathrm{T}=100 \mathrm{inPC1}$,

Table 3A

TRIVARIATE $V A R(3)$

Monte Carlo Means of Estimated Roots of M; $\mathrm{T}=100$, i.i.d. Normal Errors

\begin{tabular}{||r|r|r|r||r|r|c||}
\hline \hline & \multicolumn{3}{|c||}{ Metric of $I_{q}$} & \multicolumn{3}{c||}{ Metric of $\Sigma$} \\
\hline Roots & 1 & 2 & 3 & 1 & 2 & 3 \\
\hline PC1 & 3.782 & 0.207 & 0.010 & 15.343 & 0.462 & 0.014 \\
\hline PC2 & 16.710 & 0.056 & 0.004 & 52.091 & 0.081 & 0.008 \\
\hline PC3 & 5.075 & 0.241 & 0.010 & 18.041 & 0.439 & 0.018 \\
\hline PC4 & 0.956 & 0.064 & 0.005 & 2.507 & 0.151 & 0.012 \\
\hline PC5 & 10.986 & 0.076 & 0.007 & 31.113 & 0.157 & 0.014 \\
\hline PC6 & 4.856 & 0.149 & 0.011 & 7.023 & 0.801 & 0.017 \\
\hline \hline
\end{tabular}

the ratio $\lambda_{2} / \lambda_{3}$ is 20.7 when the identity metric is used, but 33 when the metric of $\Sigma$ is used. On the other hand, for PC5 the relevant ratios are 10.85 and 11.21, 
respectively. The same comments apply for the ratio $\lambda_{1} / \lambda_{2}$ for $\mathrm{PC} 2, \mathrm{PC} 4$, and PC6. The separation is, of course, enhanced when $T=500$ is used.

Examining the relative bias (RB) ${ }^{6}$ of the non-zero roots we find that it fluctuates across PC, but is relatively stable in the two metrics: those configurations with big stationary roots seem to be impacted more. For example, for $\mathrm{PC} 1, \lambda_{1}$

Table 3B

TRIVARIATE $V A R(3)$

Monte Carlo Means of Estimated Roots of M;

$\mathrm{T}=500$, i.i.d. Normal Errors

\begin{tabular}{||r|r|r|r||r|r|c||}
\hline \hline & \multicolumn{3}{|c||}{ Metric of $I_{q}$} & \multicolumn{3}{c||}{ Metric of $\Sigma$} \\
\hline Roots & \multicolumn{1}{|c|}{1} & 2 & 3 & 1 & 2 & 3 \\
\hline PC1 & 2.719 & 0.165 & 0.002 & 11.028 & 0.350 & 0.002 \\
\hline PC2 & 12.316 & 0.008 & 0.000 & 37.287 & 0.012 & 0.001 \\
\hline PC3 & 2.211 & 0.163 & 0.002 & 9.724 & 0.261 & 0.003 \\
\hline PC4 & 0.119 & 0.015 & 0.001 & 0.916 & 0.015 & 0.002 \\
\hline PC5 & 6.366 & 0.026 & 0.002 & 19.249 & 0.071 & 0.003 \\
\hline PC6 & 0.227 & 0.048 & 0.001 & 1.835 & 0.061 & 0.002 \\
\hline \hline
\end{tabular}

and $\mathrm{T}=100$, we have $\mathrm{RBI}=43 \%$ and $\mathrm{RBS}=45 \%$, while the relevant measures for $\mathrm{PC} 5$, which has big stationary roots, are $\mathrm{RBI}=97 \%$ and $\mathrm{RBS}=81 \%$. The respective values for $\mathrm{T}=500$ are: for $\mathrm{PC} 1, \mathrm{RBI}=2.62 \%$ and $\mathrm{RBS}=4.01 \%$; for PC5, RBI $=13.6 \%$ and $\mathrm{RBS}=12.8 \%$. This is a reduction of about $80 \%$ to $95 \%$. For the zero roots a similar measure, $\lambda_{100} / \lambda_{500}$ across samples, indicates that the reduction in the magnitude of the estimated roots is similarly quite high.

In Table 4 we have the estimated roots of J's ML procedure. The separation of the non-zero from the zero roots is, by construction, inferior to that of the roots of $\mathrm{M}$; the roots of $\mathrm{J}$ are constrained to lie in the unit interval, thus the separation is obscured. A few examples: for $\mathrm{PC} 1$ and $\mathrm{T}=100$ the ratio $\lambda_{2} / \lambda_{3}$ is 14.41 compared to 20.7 and 33 in the conformity framework. For $\mathrm{T}=500$, the relevant numbers are 78 for $\mathrm{J}$, and 82.5 and 175 for conformity. For PC5

\footnotetext{
${ }^{6}$ We define RB as the ratio of the estimated root to the true root minus one; RBI stands for the relative bias of the estimated roots in the identity metric, while RBS stands for the relative bias of the estimated roots in the error covariance matrix metric.
} 
the separation is closer to the conformity framework; for $\mathrm{T}=100$ the ratios now are 7.9 for $\mathrm{J}$ and $10.82,11.21$ for conformity, while for $\mathrm{T}=500$ are 24.5 for $\mathrm{J}$, and 13, 23.66 for conformity. Note how the absolute magnitude of the roots of $\mathrm{J}$ diminishes as the sample size increases. Also, the ratio $\lambda_{100} / \lambda_{500}$ is approximately 1.4 for the non-zero roots and between 5 and 9 for the estimated zero roots.

Table 4

TRIVARIATE $V A R(3)$

Monte Carlo Means of Estimated Roots of J; $\mathrm{T}=100 \& \mathrm{~T}=500$, i.i.d. Normal Errors

\begin{tabular}{||r|c|c|c||c|c|c||}
\hline & \multicolumn{3}{|c||}{$\mathrm{T}=100$} & \multicolumn{3}{c||}{$\mathrm{T}=500$} \\
\hline Roots & 1 & 2 & 3 & 1 & 2 & 3 \\
\hline PC1 & 0.311 & 0.173 & 0.012 & 0.274 & 0.156 & 0.002 \\
\hline PC2 & 0.303 & 0.058 & 0.007 & 0.269 & 0.011 & 0.001 \\
\hline PC3 & 0.228 & 0.131 & 0.013 & 0.164 & 0.110 & 0.002 \\
\hline PC4 & 0.189 & 0.063 & 0.008 & 0.137 & 0.011 & 0.001 \\
\hline PC5 & 0.256 & 0.079 & 0.010 & 0.209 & 0.049 & 0.002 \\
\hline PC6 & 0.353 & 0.072 & 0.009 & 0.319 & 0.012 & 0.001 \\
\hline \hline
\end{tabular}

We next turn to the examination of the quadrivariate VAR(4) system. In Tables $5 \mathrm{~A}$ and $5 \mathrm{~B}$, below, we have the Monte Carlo means of the conformity approach. The separation and bias results are now somewhat more complex. We begin by looking on the first three $\mathrm{PC}$, those with small stationary roots.

For $\mathrm{T}=100$, the $\mathrm{RB}$ measures for the estimated non-zero roots are lower for the estimators in the metric of $\Sigma$, for the first root. For PC1, we have RBI = $195 \%$, against $\mathrm{RBS}=55 \%$; for $\mathrm{PC} 2, \mathrm{RBI}=80 \%$ and $\mathrm{RBS}=45 \%$; and for $\mathrm{PC} 3$, we have RBI $=137 \%$ and RBS $=48 \%$. For the estimation of the second and third non-zero roots the RB measures for estimators in the identity metric are lower. The separation of roots is similar for both metrics, the only exception being PC3 where the ratio $\lambda_{1} / \lambda_{2}$ is 27.1 for the identity metric, but 262.7 for the metric of $\Sigma$. The same patterns are present for $T=500$ as well. For PC 4 and 5 , both of which have big stationary roots, the largest root is estimated with substantially lower $\mathrm{RB}$, even for $\mathrm{T}=100$, in both metrics than is the case for the first three PC. The relevant numbers are RBI $=70 \%$ and $\mathrm{RBS}=37 \%$ for $\mathrm{PC} 4$, and $\mathrm{RBI}=60 \%$ and $\mathrm{RBS}=31 \%$ for $\mathrm{PC} 5$. The separation of roots is again similar in both metrics, and the bias reduction across samples is now consistently very high. 
Table 5A

QUADRIVARIATE $V A R(4)$

Monte Carlo Means of Estimated Roots of M;

$\mathrm{T}=100$, i.i.d. Normal Errors

\begin{tabular}{||c|c|c|c|c||r|c|c|c||}
\hline \hline & \multicolumn{4}{|c||}{ Metric of $I_{q}$} & \multicolumn{4}{c||}{ Metric of $\Sigma$} \\
\hline Roots & 1 & 2 & 3 & \multicolumn{1}{|c||}{4} & 1 & 2 & 3 & 4 \\
\hline PC1 & 54.24 & 0.753 & 0.135 & 0.006 & 543 & 0.850 & 0.321 & 0.014 \\
\hline PC2 & 15.23 & 0.410 & 0.046 & 0.005 & 191.6 & 0.558 & 0.092 & 0.009 \\
\hline PC3 & 5.858 & 0.216 & 0.036 & 0.004 & 77.7 & 0.296 & 0.073 & 0.009 \\
\hline PC4 & 99.17 & 1.070 & 0.076 & 0.009 & 1,660 & 1.563 & 0.149 & 0.014 \\
\hline PC5 & 3.356 & 0.249 & 0.055 & 0.007 & 34.36 & 0.484 & 0.120 & 0.014 \\
\hline PC6 & 58.44 & 0.514 & 0.066 & 0.005 & 285.2 & 0.747 & 0.171 & 0.017 \\
\hline PC7 & 13.29 & 0.286 & 0.053 & 0.005 & 99.52 & 0.502 & 0.116 & 0.012 \\
\hline PC8 & 83.21 & 0.371 & 0.056 & 0.006 & 562.7 & 0.658 & 0.133 & 0.014 \\
\hline \hline
\end{tabular}

Table 5B

QUADRIVARIATE $V A R(4)$

Monte Carlo Means of Estimated Roots of M;

$\mathrm{T}=500$, i.i.d. Normal Errors

\begin{tabular}{||r|r|r|c|c||r|c|c|c||}
\hline \hline & \multicolumn{4}{|c||}{ Metric of $I_{q}$} & \multicolumn{4}{c||}{ Metric of $\Sigma$} \\
\hline Roots & 1 & 2 & 3 & \multicolumn{1}{|c||}{4} & 1 & 2 & 3 & 4 \\
\hline PC1 & 24.75 & 0.607 & 0.132 & 0.002 & 378.18 & 0.466 & 0.259 & 0.002 \\
\hline PC2 & 9.46 & 0.258 & 0.009 & 0.001 & 141.70 & 0.322 & 0.012 & 0.001 \\
\hline PC3 & 2.96 & 0.030 & 0.006 & 0.001 & 56.42 & 0.026 & 0.009 & 0.001 \\
\hline PC4 & 63.46 & 0.419 & 0.024 & 0.002 & 1,273 & 0.443 & 0.039 & 0.003 \\
\hline PC5 & 2.228 & 0.050 & 0.010 & 0.001 & 27.41 & 0.080 & 0.013 & 0.002 \\
\hline PC6 & 15.82 & 0.317 & 0.047 & 0.001 & 137.53 & 0.290 & 0.081 & 0.003 \\
\hline PC7 & 5.101 & 0.049 & 0.012 & 0.001 & 57.84 & 0.099 & 0.013 & 0.002 \\
\hline PC8 & 27.72 & 0.027 & 0.006 & 0.001 & 291.98 & 0.029 & 0.010 & 0.001 \\
\hline \hline
\end{tabular}

Finally, for PC 6 to 8 , with medium stationary roots, the results are similar to the first three PC: the largest root is estimated with substantial RB, while the other positive roots have comparably lower RB. The metric of $\Sigma$ gives lower $\mathrm{RBI}$ in estimating the largest root; for example, the relevant numbers for PC6, 
$\mathrm{T}=100$ are, $\mathrm{RBI}=488 \%$ and $\mathrm{RBS}=142 \%$. For $\mathrm{PC} 7$ we have, again for $\mathrm{T}$ $=100, \mathrm{RBI}=228 \%$ and $\mathrm{RBS}=90 \%$, respectively. The other non-zero roots have, in general, smaller RB in estimation. The bias reduction across samples, for both zero and non-zero roots, is still quite substantial but exhibits greater variablility.

Table 6

QUADRIVARIATE $V A R(4)$

Monte Carlo Means of Estimated Roots of J; $\mathrm{T}=100 \& \mathrm{~T}=500$, i.i.d. Normal Errors

\begin{tabular}{||c|c|c|c|c||c|c|c|c||}
\hline \hline & \multicolumn{5}{|c||}{$\mathrm{T}=100$} & \multicolumn{4}{c||}{$\mathrm{T}=500$} \\
\hline Roots & 1 & 2 & 3 & 4 & 1 & 2 & 3 & 4 \\
\hline PC1 & 0.395 & 0.203 & 0.121 & 0.011 & 0.353 & 0.151 & 0.114 & 0.002 \\
\hline PC2 & 0.525 & 0.192 & 0.059 & 0.008 & 0.491 & 0.146 & 0.011 & 0.001 \\
\hline PC3 & 0.525 & 0.118 & 0.048 & 0.007 & 0.493 & 0.022 & 0.008 & 0.001 \\
\hline PC4 & 0.712 & 0.156 & 0.066 & 0.010 & 0.707 & 0.086 & 0.029 & 0.002 \\
\hline PC5 & 0.779 & 0.144 & 0.063 & 0.009 & 0.786 & 0.053 & 0.012 & 0.001 \\
\hline PC6 & 0.304 & 0.164 & 0.080 & 0.011 & 0.235 & 0.098 & 0.058 & 0.002 \\
\hline PC7 & 0.395 & 0.141 & 0.059 & 0.008 & 0.349 & 0.058 & 0.011 & 0.001 \\
\hline PC8 & 0.369 & 0.143 & 0.061 & 0.009 & 0.314 & 0.022 & 0.009 & 0.001 \\
\hline \hline
\end{tabular}

The relevant results for J's procedure are given in Table 6, above. The roots exhibit the same basic behavior as in the smaller, trivariate, system. We can note in addition that the largest root does not diminish considerably, in absolute magnitude, across samples, while the other positive roots in most cases do. The ratio for the estimated zero roots is 5 to 8 , across PCs.

\subsection{Correlated and Non-Symmetric Errors}

In this subsection we present the estimated roots of the Conformity and LR frameworks when the DGP has correlated and non-symmetric errors. For these extensions, due to computatinal limitations, we used only 4 out of $6 \mathrm{PC}$ for the trivariate system, and 4 out of $8 \mathrm{PC}$ for the quadrivatiate system. Specifically, we use PC 1,2,5,6 for the trivariate system, and PC 1,2, 4, 5 for the quadrivariate. Our choices were motivated by the desire to provide some insight into the consequences of error misspecification, and the sensitivity of tests for this type of misspecification. In view of the earlier results, we report only the estimated roots of $\mathrm{M}$ in the metric of $\Sigma$. Tables 7 and 8 contain the relevant 
results for the trivariate system only. Results for the quadrivariate specification are similar and are omitted in the interest of conserving space. They are, however, available on request from the authors.

Table 7A

TRIVARIATE $V A R(3)$

Monte Carlo Means of Estimated Roots of M;

Metric of $\Sigma, \mathrm{MA}(3)$ errors

\begin{tabular}{||r|c|c|c||r|c|c||}
\hline \hline & \multicolumn{3}{|c||}{$\mathrm{T}=100$} & \multicolumn{3}{c||}{$\mathrm{T}=500$} \\
\hline Roots & 1 & 2 & 3 & 1 & 2 & 3 \\
\hline PC1 & 176.019 & 4.136 & 0.016 & 156.171 & 3.852 & 0.003 \\
\hline PC2 & 378.215 & 0.315 & 0.010 & 341.640 & 0.048 & 0.002 \\
\hline PC5 & 84.083 & 0.123 & 0.018 & 76.295 & 0.046 & 0.005 \\
\hline PC6 & 13.701 & 1.321 & 0.011 & 3.143 & 0.273 & 0.002 \\
\hline
\end{tabular}

The most striking result of the experiment reported in Table 7A is the high bias with which the non-zero roots are estimated; this bias diminishes at the rate of approximately $10-13 \%$, as the sample increases from $\mathrm{T}=100$ to $\mathrm{T}=500$. The separation of the zero from the non-zero roots remains intact; however, the zero roots are not estimated very well. For example, for $\mathrm{T}=100$, the second estimated root of $\mathrm{PC} 2$ is 0.315 . For $\mathrm{T}=500$ the second estimated root of $\mathrm{PC} 6$ is 0.273 . We recall that in the case of i.i.d. normal errors the bias of zero root estimators was of the order of $10^{-2}$; now it is of the order of $10^{-1}$.

Table 7B

TRIVARIATE $V A R(3)$

Monte Carlo Means of Estimated Roots of M;

Metric of $\Sigma$, Centered $\chi^{2}$ Errors

\begin{tabular}{||r|r|r|c||r|c|c||}
\hline \hline & \multicolumn{3}{|c||}{$\mathrm{T}=100$} & \multicolumn{3}{c||}{$\mathrm{T}=500$} \\
\hline Roots & 1 & 2 & 3 & 1 & 2 & 3 \\
\hline PC1 & 2.962 & 0.386 & 0.014 & 2.063 & 0.290 & 0.002 \\
\hline PC2 & 27.474 & 0.076 & 0.008 & 17.375 & 0.011 & 0.001 \\
\hline PC5 & 15.475 & 0.109 & 0.012 & 8.822 & 0.032 & 0.002 \\
\hline PC6 & 6.968 & 0.213 & 0.016 & 0.276 & 0.060 & 0.002 \\
\hline \hline
\end{tabular}


Table $8 \mathrm{~A}$

TRIVARIATE $V A R(3)$

Monte Carlo Means of Estimated Roots of J; MA(3) errors

\begin{tabular}{||r|c|c||c||c|c|c||}
\hline \hline & \multicolumn{3}{|c||}{$T=100$} & \multicolumn{3}{c||}{$\mathrm{T}=500$} \\
\hline Roots & 1 & 2 & 3 & 1 & 2 & 3 \\
\hline PC1 & 0.600 & 0.421 & 0.013 & 0.585 & 0.418 & 0.003 \\
\hline PC2 & 0.576 & 0.097 & 0.008 & 0.567 & 0.029 & 0.002 \\
\hline PC5 & 0.677 & 0.072 & 0.014 & 8.671 & 0.038 & 0.004 \\
\hline PC6 & 0.369 & 0.106 & 0.008 & 0.342 & 0.024 & 0.002 \\
\hline \hline
\end{tabular}

The most striking phenomenon rreported in Table 7B is the opposite of the phenomenon noted in the case of i.i.d. but non-symmetric errors. The non-zero roots are now persistently underestimated in both sample sizes. Moreover, the situation worsens as the sample increases. Exception is PC6 where the first root is overestimated, for $T=100$, but underestimated for $T=500$. Given the fact of underestimation, the separation of the roots is diminished, but the zero roots are now estimated in a similar fashion with the baseline experiments. With the exception of the second root on PC6 for a sample of 100, all the estimated zero roots have bias of order $10^{-2}$.

In Tables $8 \mathrm{~A}$ and $8 \mathrm{~B}$ we have the relevant roots for J's framework. Here, we can only make comparisons of estimated roots across experiments. For the case of MA(3) errors we also observe increases in the magnitude of the estimated non-zero roots. For example, in $\mathrm{PCl}$ and for $\mathrm{T}=100$, the estimated first and second largest roots double in magnitude, while the estimated zero root has the same magnitude. This increased magnitude remains in the larger sample, with a negligible reduction of about $1-2 \%$. On the other hand, for all estimated zero roots we can see, as in the example before, that they do not change magnitude in any substantive way from the case of i.i.d. normal errors.

For the case of the $\chi^{2}$ errors, in Table $8 \mathrm{~B}$, we have a similar situation to the encountered in Table $7 \mathrm{~B}$ (conformity framework), viz. underestimation of the non-zero roots, here of course relative to the estimated roots of the baseline case not to any 'true' roots. But this underestimation is very 'mild' in all cases except PC6. For example, in PC1 and for $\mathrm{T}=100$, the first two roots of the baseline case are higher in magnitude by about $10 \%$ and $6 \%$, respectively. For the other $\mathrm{PC}$, and for the larger sample, the relevant percentages are somewhat higher. As before, the estimated zero roots have the same magnitude as in the case of i.i.d. normal errors. 
Table 8B

TRIVARIATE $V A R(3)$

Monte Carlo Means of Estimated Roots of J;

Centered $\chi^{2}$ Errors

\begin{tabular}{||r|c|c|c||c|c|c||}
\hline \hline & \multicolumn{3}{|c||}{$\bar{T}=100$} & \multicolumn{3}{c||}{$\mathrm{T}=500$} \\
\hline Roots & 1 & 2 & 3 & 1 & 2 & 3 \\
\hline PC1 & 0.282 & 0.163 & 0.011 & 0.242 & 0.147 & 0.002 \\
\hline PC2 & 0.262 & 0.057 & 0.007 & 0.222 & 0.011 & 0.001 \\
\hline PC5 & 0.235 & 0.064 & 0.010 & 0.187 & 0.027 & 0.002 \\
\hline PC6 & 0.179 & 0.066 & 0.009 & 0.124 & 0.012 & 0.001 \\
\hline \hline
\end{tabular}

So, thus far we have seen two different patterns of behavior for the non-zero roots for both the Conformity and the LR frameworks: the presence of correlated errors leads to overestimation, while the presence of non-symmetric errors leads to underestimation. The estimated zero roots have almost the same magnitude of estimation independently of the error process. Finally, a noteworthy result from the quadrivariate system is that in the case of correlated errors the largest estimated root, in the LR context, is very close to unity for all four PC examined, as illustrated in Table $8 \mathrm{C}$ below.

Table $8 \mathrm{C}$

Estimated Largest Root of $\mathrm{J}$ Quadrivariate $V A R(4) \mathrm{MA}(4)$ Errors

\begin{tabular}{||c|c|c|c|c||}
\hline \hline $\mathrm{T}$ & PC1 & PC2 & PC4 & PC5 \\
\hline 100 & 0.991 & 0.982 & 0.917 & 0.866 \\
\hline 500 & 0.991 & 0.989 & 0.956 & 0.847 \\
\hline \hline
\end{tabular}

From Eq. (26) we see that an approximate unit root implies that there exists a nonnull vector, say $\alpha$ such that

$$
W^{\prime}\left[I_{q}-V\left(V^{\prime} V\right)^{-1} V^{\prime}\right] W \alpha \approx 0 .
$$

In turn, this means that the matrix of second moments of the residuals in the regression of $N \Delta X_{t}^{\prime}$. on $N X_{t-1}^{\prime}$. is nearly singular; this would further imply that the two vectors are nearly linearly dependent which, on the face of it, is an anomalous situation. 


\section{Size and Power of the Test Statistics}

In this section we discuss the size and power properties of the various test procedures examined earlier. First, we examine the performance characteristics of the $C R T_{Z}\left(r_{0}\right), L R T\left(r_{0}\right)$, and SW's $q_{f}(k, m)$ tests. Second, we examine the performance of the standard CRT test, all using a design where the error process is zero mean, i.i.d. and Gaussian. Finally, we examine the sensitivity of the properties of such tests to misspecification of the error process. In particular, we consider the case where the error process is a zero mean (invertible) moving average, and the case where it is centered (with one degree of freedom, i.e. it is $\chi_{1}^{2}-1$ ).

We remind the reader that $C R T_{Z}\left(r_{0}\right)$ is the conformity test based on the limiting distribution of the estimators of zero roots, while CRT is the conformity test based on the limiting distribution of estimators of non-zero roots.

\subsection{Normal Errors (i.i.d.)}

In this part we present the empirical rejection frequencies (ERF), for the various tests applied to the standard specification with a zero mean i.i.d. Gaussian process. The nominal size examined is $\alpha=0.05$. The results for the $C R T_{Z}$ and LRT tests are presented together, while the results for the $q_{f}(k, m)$ tests are presented in separate tables. Here, we present only the results for the 'trace' tests, but not those corresponding to the maximal root. The latter are presented in the Appedix, Tables A1 through A12, and we will, in the course of our discussion, comment on them when appropriate.

Tables 9 and 10 contain the results for the trivariate system, while Tables 11 and 12 contain those for the quadrivariate system.

The tables are meant to be read as follows: for the $C R T_{Z}$ and $L R T$ tests, each column block has the ERF for the hypothesis that the last $r_{0}$ roots are zero. So, for example, in Table 9A the first two columns have the ERF for the hypothesis that all three roots are zero, which is the hypothesis of no cointegration; the next two columns have the ERF for the hypothesis that the smallest two roots are zero, thus for the hypothesis that the cointegrating rank is 1 .

In the tables containing the ERF for the SW tests $q_{f}(k, m), k$ is set equal to $q$ and each column has the ERF for the hypothesis that the largest root is one $(m=1)$, the second largest root is one $(m=2)$, the third largest root is one $(m=3)$ and so on.

In the SW framework, a rejection under the column labeled $m=1$ indicates a rejection of the null that the largest root is one and, consequently, that the sequence is stationary and thus there are no "common trends"; a rejection under the column labeled $m=2$ means a rejection of the null that the second 
largest root is one and, consequently, that the system is cointegrated of rank (at least) two, or there is at most one "common trend"; finally, a rejection under the column $m=3$ indicates a rejection of the null that the third largest root is one and, consequently, that the system is cointegrated of rank (at least) one, or there are at most two "common trends", and so on.

Finally, we should note that our use of the SW test is not precisely that intended by the authors in their notation $q_{f}(k, m)$. None the less our test implementation is based on the limiting distribution of the ordered roots as given in their paper.

Table $9 \mathrm{~A}$ below contains the ERF for the trivariate system with $\mathrm{T}=100$. Both the $C R T_{Z}(3)$ and $L R T(3)$ tests strongly reject no cointegration, except for the $L R T(3)$ in $\mathrm{PC} 4$ where it accepts no cointegration, i.e. that there are three unit roots $42 \%$ of the time. Since PC4 has only one unit root and big stationary roots, it appears that the imperfect separation of roots in the LR framework leads to considerable loss of power. The tests for $r_{0}=2$ show that the two tests exhibit a tradeoff between size and power. Thus, for PC1 and PC2 they behave almost identically, with correct sizes and power. For PC3 (one unit

Table $9 \mathrm{~A}$

\section{TRIVARIATE $V A R(3)$}

Empirical Rejection Frequencies, $C R T_{Z}\left(r_{0}\right), L R T\left(r_{0}\right)$

$\mathrm{T}=100$, Nominal Size 0.05, i.i.d. Normal Errors

\begin{tabular}{||r|r|r||r|r||r|r||}
\hline \hline Test & $C R T_{Z}(3)$ & LRT (3) & $C R T_{Z}(2)$ & LRT(2) & $C R T_{Z}(1)$ & LRT(1) \\
\hline PC1 & 1.000 & 1.000 & 0.983 & 0.932 & 0.005 & 0.047 \\
\hline PC2 & 1.000 & 0.985 & 0.065 & 0.054 & 0.000 & 0.006 \\
\hline PC3 & 1.000 & 0.981 & 0.932 & 0.693 & 0.011 & 0.060 \\
\hline PC4 & 0.999 & 0.580 & 0.319 & 0.072 & 0.000 & 0.013 \\
\hline PC5 & 1.000 & 0.952 & 0.343 & 0.207 & 0.003 & 0.039 \\
\hline PC6 & 1.000 & 0.994 & 0.851 & 0.136 & 0.011 & 0.019 \\
\hline \hline
\end{tabular}

root) the conformity test has considerably more power than the LR test; for PC4 and PC6 the conformity test exhibits considerable size distortions, while the size distortions of the LR test are small by comparison. This is perhaps not entirely unexpected in view of the fact that these two PC have a "nearly collinear" parametric structure, and the conformity test is based directly on the rank of $\Pi(1),(\operatorname{rank}(M)=\operatorname{rank}[(\Pi(1)])$. In connection with these results, it worth noting that the maximal root test for $r_{0}=3$ for PC3 shows that the conformity test has an ERF of 1.0, while the LR test has an ERF of .855 . 
Finally, we observe from the last two columns of the table that both tests have comprable ERF in detecting cointegration, in that the entries of the last column (which relates to the test that the smallest root of the system is zero) are very close to zero, and comparable as between the two tests.

In Table 9B, below, we give the ERF for the trivariate system when $\mathrm{T}=500$. As expected, the results closely parallel those indicated by the asympotic theory, and the problems encountered with the smaller sample disappear, in all but one instance: The $C R T_{Z}(2)$ test for the last two roots in PC6 still exhibits a high size distortion.

The results for SW's $q_{f}(k, m)$ test are given in Table 10 below. They show that this test is very weak and inconclusive; it suffers both from power problems and size distortions. These problems do not seem to disappear when the sample is increased to $\mathrm{T}=500$. As an example, for $\mathrm{T}=100, \mathrm{PC} 1$ (which has one unit root), the test correctly rejects the hypothesis that the third and second largest roots are one, (for $m=2$ and $m=3$ ), but it also rejects the hypothesis that the largest root is one in $63 \%$ of the replications, and thus finds stationarity!

Table 9B

TRIVARIATE $V A R(3)$

Empirical Rejection Frequencies, $C R T_{Z}\left(r_{0}\right), \operatorname{LRT}\left(r_{0}\right)$

$\mathrm{T}=500$, Nominal Size 0.05, i.i.d. Normal Errors

\begin{tabular}{||r|r|r||r|r||r|r||}
\hline \hline Test & \multicolumn{1}{|c|}{$C R T_{Z}(3)$} & LRT $(3)$ & $C R T_{Z}(2)$ & LRT $(2)$ & $C R T_{Z}(1)$ & LRT(1) \\
\hline PC1 & 1.000 & 1.000 & 1.000 & 1.000 & 0.001 & 0.045 \\
\hline PC2 & 1.000 & 1.000 & 0.016 & 0.050 & 0.000 & 0.007 \\
\hline PC3 & 1.000 & 1.000 & 1.000 & 1.000 & 0.002 & 0.062 \\
\hline PC4 & 1.000 & 1.000 & 0.057 & 0.071 & 0.000 & 0.011 \\
\hline PC5 & 1.000 & 1.000 & 0.946 & 0.985 & 0.002 & 0.063 \\
\hline PC6 & 1.000 & 1.000 & 0.445 & 0.071 & 0.000 & 0.007 \\
\hline \hline
\end{tabular}

This problem is incountered among all configurations, and in both samples. As another example, consider PC4 (which has two unit roots) where the test almost always accepts the null that the third largest root is one, i.e. that the sequence is $I(1)$ and not cointegrated. By constrast, for $\mathrm{T}=100$ in $37 \%$ of the replications it accepts the view that the sequence is stationary, since it rejects the null that the largest root is one. For $\mathrm{T}=500$ this situation occurs in $59 \%$ of the replications. 
Thus, our conclusion has to be that the SW test procedure does not provide a reliable guide for empirical research, a conclusion also reached in Yap and Reinsel (1995).

Table 10

\section{TRIVARIATE $V A R(3)$}

Empirical Rejection Frequencies for S \& W's $q_{f}(k, m)$ test $k=3$, Nominal Size 0.05 , i.i.d. Normal Errors

\begin{tabular}{||c|c|c|c||c|c|c||}
\hline \hline & \multicolumn{3}{|c||}{$\mathrm{T}=100$} & \multicolumn{3}{c||}{$\mathrm{T}=500$} \\
\hline$m \rightarrow$ & 1 & 2 & 3 & 1 & 2 & 3 \\
\hline PC1 & 0.637 & 0.995 & 0.983 & 0.566 & 1.000 & 1.000 \\
\hline PC2 & 0.528 & 0.377 & 0.530 & 0.490 & 0.354 & 1.000 \\
\hline PC3 & 0.425 & 0.086 & 0.129 & 0.696 & 0.953 & 0.999 \\
\hline PC4 & 0.377 & 0.006 & 0.002 & 0.561 & 0.593 & 0.004 \\
\hline PC5 & 0.353 & 0.172 & 0.293 & 0.424 & 0.383 & 0.996 \\
\hline PC6 & 0.146 & 0.013 & 0.053 & 0.619 & 0.173 & 0.070 \\
\hline \hline
\end{tabular}

In Tables 11 and 12 we present the results for the quadrivariate model; Table $11 \mathrm{~A}$ reports ERF for the conformity and LR tests for sample size $\mathrm{T}=100$, Table 11B those for $\mathrm{T}=500$, while Table 12 contains the results for the SW tests.

Both conformity and LR tests detect cointegration quite well although the conformity test exhibits more power as evident from the first two columns of the table. The tests for the last 3 roots, columns labeled $C R T_{Z}(3)$ and $L R T(3)$, respectively, show the same pattern of trade-off between size and power as we noted in the trivariate system.

For $\mathrm{PC} 1$ both tests correctly reject the hypothesis that all four roots are zero. For PC2 $C R T_{Z}(3)$ has considerable power, .95, while the $L R T(3)$ test has relatively low power, .587 , i.e. in approximately $42 \%$ of the replications it accepts the hypothesis the last three roots are zero, i.e. that the system has three unit roots, while in truth $\mathrm{PC} 2$ has only two unit roots. On the other hand for PC3 both tests have size problems; $C R T_{Z}(3)$ rejects the hypothesis that the last three roots are zero in about $50 \%$ of the replications, while $L R T(3)$ rejects this hypothesis in about $10 \%$ of the replications, even though the nominal size is $5 \%$. In PC4 and PC5, which both have large stationary roots, we see that $L R T(3)$ has severe power problems: in about $60 \%$ and $70 \%$ of the replications it accepts the hypothesis that the last three roots are zero, i.e. it accepts the 
hypothesis that the cointegrating rank is 1 , instead of the correct 3 and 2 , respectively.

Table $11 \mathrm{~A}$

QUADRIVARIATE $V A R(4)$

Empirical Rejection Frequencies

$C R T_{Z}\left(r_{0}\right), \operatorname{LRT}\left(r_{0}\right), \mathrm{T}=100$

Nominal Size 0.05, i.i.d. Normal Errors

\begin{tabular}{||r|r|r||r|r||}
\hline \hline Test & $C_{Z}(4)$ & LRT(4) & $C R T_{Z}(3)$ & LRT(3) \\
\hline PC1 & 1.00 & 1.000 & 1.000 & 0.943 \\
\hline PC2 & 1.00 & 1.000 & 0.950 & 0.587 \\
\hline PC3 & 1.00 & 1.000 & 0.502 & 0.105 \\
\hline PC4 & 1.00 & 1.000 & 0.989 & 0.400 \\
\hline PC5 & 1.00 & 1.000 & 0.839 & 0.318 \\
\hline PC6 & 1.00 & 0.981 & 0.984 & 0.568 \\
\hline PC7 & 1.00 & 0.994 & 0.847 & 0.260 \\
\hline PC8 & 1.00 & 0.986 & 0.892 & 0.295 \\
\hline & & & & \\
\hline \hline Test & $C R T_{Z}(2)$ & LRT(2) & $C R T_{Z}(1)$ & LRT(1) \\
\hline \hline PC1 & 0.743 & 0.582 & 0.003 & 0.044 \\
\hline PC2 & 0.048 & 0.061 & 0.000 & 0.010 \\
\hline PC3 & 0.011 & 0.014 & 0.000 & 0.007 \\
\hline PC4 & 0.194 & 0.074 & 0.001 & 0.015 \\
\hline PC5 & 0.100 & 0.066 & 0.000 & 0.020 \\
\hline PC6 & 0.245 & 0.152 & 0.001 & 0.036 \\
\hline PC7 & 0.093 & 0.045 & 0.000 & 0.014 \\
\hline PC8 & 0.146 & 0.057 & 0.003 & 0.022 \\
\hline \hline
\end{tabular}

The $C R T_{Z}(3)$ test exhibits very serious size distortions for PC8; in $89 \%$ of the replications it rejects the (true) null that the last three roots are zero. The comparable figure for the $L R T(3)$ is $30 \%$, so that it too suffers size distortions albeit of a milder nature. For PC7 (which has only two unit roots) $C R T_{Z}(3)$ has considerably more power than the $L R T(3)$, since the latter accepts the hypothesis that the cointegrating rank is $1\left(q-r_{0}\right)$ instead of the correct 2 in $74 \%$ of the replications. By contrast, the $C R T_{Z}(3)$ test does so in only $15 \%$ of the replications. For the last two roots (columns labeled $C R T_{Z}(2), C R T_{Z}(1)$, and similarly for the LR test) we observe relatively low power for both tests 
even though on balance the conformity tests is the more powerful of the two; the size characteristics are also similar with the LR test having empirical size somewhat closer to the nominal one.

Table 11B

QUADRIVARIATE $V A R(4)$

Empirical Rejection Frequencies

$C R T_{Z}\left(r_{0}\right), \operatorname{LRT}\left(r_{0}\right), \mathrm{T}=500$

Nominal Size 0.05, i.i.d. Normal Errors

\begin{tabular}{||r|r|r||r|r||}
\hline \hline Test & $C R T_{\mathcal{Z}}(4)$ & LRT(4) & $C R T_{Z}(3)$ & LRT $(3)$ \\
\hline PC1 & 1.00 & 1.00 & 1.000 & 1.000 \\
\hline PC2 & 1.00 & 1.00 & 1.000 & 1.000 \\
\hline PC3 & 1.00 & 1.00 & 0.040 & 0.057 \\
\hline PC4 & 1.00 & 1.00 & 1.000 & 1.000 \\
\hline PC5 & 1.00 & 1.00 & 0.883 & 0.895 \\
\hline PC6 & 1.00 & 1.00 & 1.000 & 1.000 \\
\hline PC7 & 1.00 & 1.00 & 0.975 & 0.955 \\
\hline PC8 & 1.00 & 1.00 & 0.096 & 0.080 \\
\hline & & & & \\
\hline \hline Test & $C R T_{Z}(2)$ & LRT(2) & $C R T_{Z}(1)$ & LRT(1) \\
\hline \hline PC1 & 1.000 & 1.000 & 0.000 & 0.052 \\
\hline PC2 & 0.003 & 0.055 & 0.000 & 0.067 \\
\hline PC3 & 0.000 & 0.003 & 0.000 & 0.002 \\
\hline PC4 & 0.301 & 0.755 & 0.001 & 0.057 \\
\hline PC5 & 0.004 & 0.071 & 0.000 & 0.007 \\
\hline PC6 & 0.979 & 1.000 & 0.001 & 0.051 \\
\hline PC7 & 0.004 & 0.051 & 0.000 & 0.012 \\
\hline PC8 & 0.001 & 0.011 & 0.000 & 0.053 \\
\hline \hline
\end{tabular}

In addition, it is worth noting that the conformity maximal root test, $M R C(2)$, for the second root has much higher power than the corresponding trace, $C R T_{Z}(2)$, test; as an example for $\mathrm{PC} 1$ the table entry for the trace test is .743; if we recorded the maximal root test based result it would have been .871 . The same is true of PC4 and PC6. The opposite phenomenon holds for the maximal root of the LR test, viz. that the power characteristics of the maximal root based LR test are lower than those of the trace based LR test.

Finally, as before, both tests easily detect the presence of cointegration. 
The results for $\mathrm{T}=500$ are given in Table 11B. As expected, both tests now have almost no size or power problems. It is noteworthy how close the results are on the tests for the last three roots (upper panel). An anomaly in terms of earlier ressults occurs in PC4 (which has one unit root and two large stationary roots), where the conformity test rejects the hypothesis that the last two roots are zero in $30 \%$ of the replications only, while the LR test rejects this hypothesis in $75 \%$ of the replications. This is the only case where the $C R T_{Z}$ test underestimates the true cointegrating rank, a feature, thus far, almost exclusively of the LRT test. Again, it is worth noting that the maximal root based conformity test in this case has higher power, .504 while the maximal root based LR test has identical power as reported in the table, viz. .747.

We conclude this section by examining the results obtained using the SW $q_{f}(k, m)$ test for the quadrivariate system. They are given in Table 12 below. As in the smaller system, we have confusing results here as well. The test's performance is very weak and inconclusive: the test correctly detects the presence of cointegration, by rejecting the null of 4 'common trends', but suffers from power losses in that it frequently accepts stationarity! In light of these findings the intermediate results about the rank are of no significance and thus we must. reiterate our finding that the SW cannot be recommended for use in empirical applications.

Table 12

\section{QUADRIVARIATE $V A R(4)$}

Empirical Rejection Frequencies for $S \&$ W's $q_{f}(k, m)$ test $k=4$, Nominal Size 0.05, i.i.d. Normal Errors

\begin{tabular}{||c|c|c|c|c||c|c|c|c||}
\hline \hline & \multicolumn{5}{|c||}{$\mathrm{T}=100$} & \multicolumn{4}{c||}{$\mathrm{T}=500$} \\
\hline$m \rightarrow$ & 1 & 2 & 3 & 4 & 1 & 2 & 3 & 4 \\
\hline PC1 & 0.879 & 0.997 & 0.999 & 0.996 & 0.905 & 1.000 & 1.000 & 1.00 \\
\hline PC2 & 0.688 & 0.720 & 0.966 & 0.997 & 0.656 & 0.697 & 1.000 & 1.00 \\
\hline PC3 & 0.613 & 0.403 & 0.615 & 0.892 & 0.599 & 0.349 & 0.878 & 1.00 \\
\hline PC4 & 0.512 & 0.183 & 0.858 & 0.999 & 0.789 & 0.450 & 1.000 & 1.00 \\
\hline PC5 & 0.375 & 0.087 & 0.157 & 0.972 & 0.465 & 0.552 & 0.366 & 1.00 \\
\hline PC6 & 0.498 & 0.340 & 0.223 & 0.978 & 0.382 & 0.997 & 0.992 & 1.00 \\
\hline PC7 & 0.332 & 0.133 & 0.565 & 0.998 & 0.359 & 0.096 & 1.000 & 1.00 \\
\hline PC8 & 0.204 & 0.045 & 0.385 & 0.974 & 0.221 & 0.111 & 0.531 & 1.00 \\
\hline \hline
\end{tabular}




\subsection{Correlated and Non-Symmetric Errors}

In this subsection we examine the sensitivity of the tests examined earlier to misspecification in the error process. In particular we examine "VARs" whose error process is a moving average, and a centered chi-square (i.e. $\chi_{1}^{2}-1$. We present, in Tables 13,14,15, and 16, results only for the conformity and LR tests. In the trivariate case we deal with $\mathrm{PC} 1, \mathrm{PC} 2, \mathrm{PC} 5$ and $\mathrm{PC} 6$, while in the quadrivariate case we deal with $\mathrm{PC} 1, \mathrm{PC} 2, \mathrm{PC} 4$, and $\mathrm{PC} 5$.

In Tables 13 through 16 we present the ERF for the case where the error process is misspecified. The entries therein, therefore, are not to be interpreted in the same strict manner as in the earlier tables; in that case the entries serve two important purposes. First, they indicate how closely the critical values obtained from the limiting distribution approximate the finite (small in the case of $\mathrm{T}=$ 100) critical values and, second, how large should the sample be in order to get a very close approximation. In addition, they also provide information on the relative performance of the conformity vis-a-vis the likelihood ratio test. In the case where the error processes are misspecified the results shed light only on the relative performance of the two tests, except in the case where the misspecified error process yields precisely the same asymptotic theory as the standard (i.i.d. normal) error process. When we use an MA(3) error process the limiting distribution of both tests is not the same. In the case of i.i.d. $\chi_{1}^{2}-1$ errors, the latter would yield the same limiting distribution theory and in principle such results should have the same interpretation as in the earlier tables.

Table 13A

TRIVARIATE $V A R(3)$

Empirical Rejection Frequencies, $C R T_{Z}\left(r_{0}\right), \operatorname{LRT}\left(r_{0}\right)$ $\mathrm{T}=100$, Nominal Size 0.05, MA(3) Errors

\begin{tabular}{||r|r|r||r|r||r|r||}
\hline \hline Test & $C_{R} T_{Z}(3)$ & LRT(3) & $C R T_{Z}(2)$ & LRT(2) & $C R T_{Z}(1)$ & LRT(1) \\
\hline PC1 & 1.00 & 1.00 & 1.000 & 1.000 & 0.013 & 0.071 \\
\hline PC2 & 1.00 & 1.00 & 0.517 & 0.327 & 0.003 & 0.019 \\
\hline PC5 & 1.00 & 1.00 & 0.187 & 0.155 & 0.004 & 0.067 \\
\hline PC6 & 1.00 & 1.00 & 0.854 & 0.381 & 0.004 & 0.016 \\
\hline \hline
\end{tabular}

In Table 13A we have the $\mathrm{ERF}$ for $\mathrm{T}=100$, with the trivariate system and moving average errors. Both tests always reject the hypothesis of nocointegration, i.e. $r_{0}=3$, as is evident from the ERF in the first column 
block of the table. For PC1 we see almost no difference relative to the case of i.i.d. normal errors, see Table 9A, except slight increases in power for $r_{0}=2$, for both tests, and a slight increase in the empirical size of both tests for the last root. For PC2 both tests have size problems when testing for the last two roots, $r_{0}=2$; the $C R T_{Z}(2)$ test suffers more with an ERF of $51.7 \%$ versus $32.7 \%$ for the LRT(2) test. In PC5 both tests have power problems when testing for the last two roots; now $L R T(2)$ suffers slightly more than $C R T_{Z}(2)$. For PC6 we have again size problems, as in $\mathrm{PC} 2$, when testing $r_{0}=2$. The $C R T_{Z}(2)$ test has higher size distortion compared to the LRT test. Note that the size distortion for the $L R T$ test, for PC6, is almost the same as its size distortion for PC2. Both PC2 and PC6 have two unit roots but they differ in the magnitude of their stationary roots. Both tests fare equally badly or equally well, except in the case of PC6 (which has two unit roots and two large stationary roots) where the conformity test rejects the null of two zero roots in $85 \%$ of the replications, while the likelihood ratio test does so in only $38 \%$ of the replications. Finally, both tests have no problem in detecting cointegration, i.e. they almost always accept that the last root is zero.

The results for $\mathrm{T}=500$, given in Table $13 \mathrm{~B}$ below, do not show substantial improvement, although now the performance of the two tests is equally bad or equally good, depending on one's point of view. For example the poor performance of the conformity test for PC6 is offset by the uniformly worse performance of the likelihood ratio test in testing whether the smallest root is zero, which is rejected inordinately frequently by that test.

Table 13B

TRIVARIATE $V A R(3)$

Empirical Rejection Frequencies, $C R T_{Z}\left(r_{0}\right), L R T\left(r_{0}\right)$

$\mathrm{T}=500$, Nominal Size 0.05, MA(3) Errors

\begin{tabular}{||r|r|r||r|r||r|r||}
\hline \hline Test & $C R T_{Z}(3)$ & LRT(3) & $C R T_{Z}(2)$ & LRT(2) & $C R T_{Z}(1)$ & LRT(1) \\
\hline PC1 & 1.00 & 1.00 & 1.000 & 1.000 & 0.010 & 0.087 \\
\hline PC2 & 1.00 & 1.00 & 0.477 & 0.548 & 0.003 & 0.052 \\
\hline PC5 & 1.00 & 1.00 & 0.874 & 0.988 & 0.030 & 0.173 \\
\hline PC6 & 1.00 & 1.00 & 0.747 & 0.415 & 0.001 & 0.022 \\
\hline \hline
\end{tabular}

The ERF of the two tests for the case of non-symmetric errors is given in Tables $14 \mathrm{~A}$ and $14 \mathrm{~B}$ below. Here the limiting distribution theory remains valid and thus the results have the same interpretation as those in Tables 10 and 11. 
Table $14 \mathrm{~A}$

TRIVARIATE $V A R(3)$

Empirical Rejection Frequencies, $C R T_{Z}\left(r_{0}\right), L R T\left(r_{0}\right)$

$\mathrm{T}=100$, Nominal Size 0.05, $\chi^{2}$ Errors

\begin{tabular}{||r|r|r||r|r||r|r||}
\hline \hline Test & \multicolumn{1}{|c|}{$C R T_{Z}(3)$} & LRT(3) & $C R T_{Z}(2)$ & LRT(2) & $C R T_{Z}(1)$ & LRT(1) \\
\hline PC1 & 1.000 & 1.000 & 0.961 & 0.893 & 0.005 & 0.051 \\
\hline PC2 & 1.000 & 0.929 & 0.054 & 0.053 & 0.000 & 0.013 \\
\hline PC5 & 1.000 & 0.868 & 0.169 & 0.099 & 0.001 & 0.023 \\
\hline PC6 & 0.999 & 0.562 & 0.654 & 0.082 & 0.005 & 0.019 \\
\hline \hline
\end{tabular}

Table 14A has results for $\mathrm{T}=100$ and Table $14 \mathrm{~B}$ has results for $\mathrm{T}=500$.

For $\mathrm{T}=100$, the first two columns indicate a superior performance on the part of the conformity test vis-a-vis the likelihood ratio test. For example in the case of PC6 (which has two unit roots and one large stationary root) the likelihood ratio test in $44 \%$ of the replications accepts the hypothesis that the sequence is $I(1)$ and noncointegrated. On the other hand, for the same PC6, $C R T_{z}$ rejects the hypothesis of two zero roots in $65 \%$ of the replications (size distortion), while the likelihood ratio test does so in only $8 \%$ of the replications. Otherwise the performance of the two tests is comparable. As we noted earlier the maximal root version of the conformity test has considerably smaller size distortion and approximately the same power. Thus, for example, if instead of the $C R T_{Z}(2)$ (trace) test we used the maximal root test (i.e. used only the second root) the ERF would have been .212 for the conformity test and .077 for the likelihood ratio test. Contrast this with Table 14A where the corresponding entries are .654 and .082 , respectively.

Table 14B

TRIVARIATE $V A R(3)$

Empirical Rejection Frequencies, $C R T_{Z}\left(r_{0}\right), \operatorname{LRT}\left(r_{0}\right)$ $\mathrm{T}=500$, Nominal Size $0.05, \chi^{2}$ Errors

\begin{tabular}{||r|r|r||r|r||r|r||}
\hline \hline Test & $C R T_{Z}(3)$ & LRT $(3)$ & $C R T_{Z}(2)$ & LRT $(2)$ & $C R T_{Z}(1)$ & LRT(1) \\
\hline PC1 & 1.00 & 1.00 & 1.000 & 1.000 & 0.001 & 0.053 \\
\hline PC2 & 1.00 & 1.00 & 0.006 & 0.045 & 0.000 & 0.004 \\
\hline PC5 & 1.00 & 1.00 & 0.385 & 0.636 & 0.001 & 0.054 \\
\hline PC6 & 1.00 & 1.00 & 0.543 & 0.071 & 0.001 & 0.006 \\
\hline \hline
\end{tabular}


The results for $\mathrm{T}=500$ are given in Table 14B. Again the two tests perform equally well, except in PC5 and PC6, when testing the hypothesis that the last two roots are zero. For PC5 the power of the conformity test is .385 , while that of the likelihood ratio test is .636. As we noted earlier, however, if we employ tests based on the maximal root the relevant powers would have been .522 and .62 , respectively. For PC6, the conformity test rejects a correct null too frequently, in $54 \%$ of the replications.

In Table 15A, below, we present the ERF for the quadrivariate system, $\mathrm{T}=$ 100 , and MA(4) errors.

In the first two columns of the table it is evident that the two tests uniformly reject the hypothesis that the sequence is $I(1)$ and not cointegrated. In the next two columns the conformity test unformly rejects the hypothesis that there are three zero roots, i.e. that the system has three unit roots. The likelihood ratio test, however, accepts the hypothesis of three zero roots in $32 \%$ of the replications, i.e. it finds cointegration of rank 1, instead of the correct rank 2. In the next two columns the conformity test exhibits size distortions, i.e. it rejects the correct null of two zero roots in PC2 and PC5 in about $78 \%$ of

Table 15A

\section{QUADRIVARIATE $V A R(4)$}

Empirical Rejection Frequencies, $C R T_{Z}\left(r_{0}\right), L R T\left(r_{0}\right)$ $\mathrm{T}=100$, Nominal Size 0.05, MA(4) Errors

\begin{tabular}{||r|r|r||r|r||}
\hline \hline Test & $C R T_{Z}(4)$ & LRT(4) & $C R T_{Z}(3)$ & LRT(3) \\
\hline PC1 & 1.00 & 1.00 & 1.00 & 1.000 \\
\hline PC2 & 1.00 & 1.00 & 1.00 & 1.000 \\
\hline PC4 & 1.00 & 1.00 & 1.00 & 1.000 \\
\hline PC5 & 1.00 & 1.00 & 1.00 & 0.685 \\
\hline & & & & \\
\hline \hline Test & $C R T_{Z}(2)$ & LRT(2) & $C R T_{Z}(1)$ & LRT(1) \\
\hline \hline PC1 & 1.000 & 1.000 & 0.006 & 0.053 \\
\hline PC2 & 0.785 & 0.058 & 0.001 & 0.004 \\
\hline PC4 & 1.000 & 0.999 & 0.132 & 0.161 \\
\hline PC5 & 0.780 & 0.174 & 0.046 & 0.033 \\
\hline \hline
\end{tabular}

the replications, while the likelihood ratio test does so in about $6 \%$ and $17 \%$ of the replications, respectively. 
Finally, in the tests of the smallest root, both conformity and likelihood ratio tests do equally well, or equally badly, exhibiting size distortions for PC4, i.e. they reject a correct null in $13 \%$ and $16 \%$ of the replications respectively.

When the sample size is increased to $\mathrm{T}=500$, the behavior of the coformity test improves, while that of the likelihood ratio test worsens. None the less size distortions persist for the conformity test in the case of PC2 and PC5 and the size distortion for the likelihood ratio test in PC5 ( $L R T(2))$ worsens.

In Tables $16 \mathrm{~A}$ and $16 \mathrm{~B}$ we presents ERF for the case when the error process is non-symmetric $\left(\chi_{1}^{2}-1\right)$. For $T=100$, the conformity test exhibits considerably more power than the likelihood ratio test, as evident from the first four columns of Table 16A. The conformity test exhibits slightly more size distortions than the likelihood ratio test, as evident from the third column.

Table 15B

QUADRIVARIATE $V A R(4)$

Empirical Rejection Frequencies, $C R T_{Z}\left(r_{0}\right), \operatorname{LRT}\left(r_{0}\right)$

$\mathrm{T}=500$, Nominal Size 0.05, MA(3) Errors

\begin{tabular}{||r|r|r||r|r||}
\hline \hline Test & $C R T_{Z}(4)$ & LRT(4) & $C R T_{Z}(3)$ & LRT $(3)$ \\
\hline PC1 & 1.00 & 1.00 & 1.00 & 1.00 \\
\hline PC2 & 1.00 & 1.00 & 1.00 & 1.00 \\
\hline PC4 & 1.00 & 1.00 & 1.00 & 1.00 \\
\hline PC5 & 1.00 & 1.00 & 1.00 & 1.00 \\
\hline & & & & \\
\hline \hline Test & $C R T_{Z}(2)$ & LRT(2) & $C R T_{Z}(1)$ & LRT $(1)$ \\
\hline \hline PC1 & 1.000 & 1.000 & 0.001 & 0.051 \\
\hline PC2 & 0.592 & 0.049 & 0.001 & 0.007 \\
\hline PC4 & 1.000 & 0.999 & 0.001 & 0.096 \\
\hline PC5 & 0.476 & 0.197 & 0.002 & 0.013 \\
\hline \hline
\end{tabular}

As we also noted in the trivariate system, the power characteristics of the conformity test is improved if we use, instead of the trace, the maximal root test. Thus, as an example, for $\mathrm{PC} 1$ and the test that the last two roots are null, the maximal root test will yield an ERF of .816, instead of .685, as recorded in the table.

The use of the larger sample size, $\mathrm{T}=500$, improves the performance of both tests, but the likelihood ratio test imrpoves more significantly. The two tests perform equally well in testing whether all four roots, the last three roots, 
Table $16 \mathrm{~A}$

QUADRIVARIATE $V A R(4)$

Empirical Rejection Frequencies, $C R T_{Z}\left(r_{0}\right), L R T\left(r_{0}\right)$ $\mathrm{T}=100$, Nominal Size $0.05, \chi^{2}$ Errors

\begin{tabular}{||r|r|r||r|r||}
\hline \hline Test & $C_{R T_{Z}(4)}$ & LRT $(4)$ & $C R T_{Z}(3)$ & LRT(3) \\
\hline PC1 & 1.00 & 0.999 & 1.000 & 0.931 \\
\hline PC2 & 1.00 & 0.999 & 0.981 & 0.720 \\
\hline PC4 & 1.00 & 0.975 & 0.999 & 0.422 \\
\hline PC5 & 1.00 & 0.955 & 0.749 & 0.259 \\
\hline & & & & \\
\hline \hline Test & $C R \bar{T}_{Z}(2)$ & LRT(2) & $C R T_{Z}(1)$ & LRT $(1)$ \\
\hline \hline PC1 & 0.685 & 0.548 & 0.001 & 0.041 \\
\hline PC2 & 0.043 & 0.063 & 0.001 & 0.010 \\
\hline PC4 & 0.173 & 0.088 & 0.001 & 0.027 \\
\hline PC5 & 0.062 & 0.055 & 0.001 & 0.019 \\
\hline \hline
\end{tabular}

Table 16B

QUADRIVARIATE $V A R(4)$

Empirical Rejection Frequencies, $C R T_{Z}\left(r_{0}\right), L R T\left(r_{0}\right)$ $\mathrm{T}=500$, Nominal Size $0.05, \chi^{2}$ Errors

\begin{tabular}{||r|r|r||r|r||}
\hline \hline Test & $C R T_{Z}(4)$ & LRT(4) & $C R T_{Z}(3)$ & LRT(3) \\
\hline PC1 & 1.00 & 1.00 & 1.000 & 1.000 \\
\hline PC2 & 1.00 & 1.00 & 1.000 & 1.000 \\
\hline PC4 & 1.00 & 1.00 & 1.000 & 1.000 \\
\hline PC5 & 1.00 & 1.00 & 0.783 & 0.811 \\
\hline & & & & \\
\hline \hline Test & $C R T_{Z}(2)$ & LRT(2) & $C R T_{Z}(1)$ & LRT(1) \\
\hline \hline PC1 & 1.000 & 1.000 & 0.001 & 0.051 \\
\hline PC2 & 0.001 & 0.049 & 0.001 & 0.010 \\
\hline PC4 & 0.200 & 0.710 & 0.001 & 0.040 \\
\hline PC5 & 0.004 & 0.073 & 0.001 & 0.016 \\
\hline \hline
\end{tabular}

or the last root, are null; in the test of whether the last two roots are null, i.e. 
that the system has two unit roots (when in fact it has only one) the conformity test has low power relative to that of the likelihood ratio test, .2 as against .71 .

We should note gain that the maximal root version of the conformity test exhibits somewhat greater power. As an example, if we employ the maximal root to test the hypothesis that the last three roots are zero for PC5 (which in fact has only two zero roots) the ERF would have been .902 , instead of .783 as recorded in Table 16B. Similarly, for PC4 the maximal root test has an ERF of .380 , instead of .200 as is recorded in Table 16B.

We conclude the discussion of the performance of the conformity test based on zero root estimators by examining the case of trivariate random walk and a 10-variate $V A R(2)$, of cointegrating rank nine and eight, i.e. with one and two unit roots, respectively. The first is designed to guage the performance of the conformity and likelihood ratio tests when the underlying sequence is $I(1)$ and not cointegrated, and the second to examine the cojecture that in reltively small samples the likelihood ratio test will do relatively less well in large systems, especially when the test pertains to the smaller roots of the system. Due to computatinal limitation we did not undertake a full examination of such structures, but Tables 17, 18, and 19 are all based on 3,500 replications. The construction of the vector random walk is self evident. In the 10-variate system the stationary roots were, respectively, $.6, .55, .5, .45, .4, .35, .3, .26, .22, .19$, $.17, .14, .12, .09, .07, .06, .04, .02$ and $.6, .55, .5, .45, .4, .35, .3, .26, .22, .19$, $.17, .14, .12, .09, .07, .06, .04$. Table 17 , contains the results for the trivariate random walk, and Tables 18 and 19 those for the 10 -variate system.

Table 17

TRIVARIATE VECTOR RANDOM WALK

ERF, Trace and Maximal Root Tests

Various Samples, Nominal Size 0.05, normal errors (i.i.d.)

\begin{tabular}{||c||r|r||r|r||}
\hline \hline $\mathrm{T}$ & \multicolumn{1}{||c|}{$\overline{R R T_{Z}}(3)$} & \multicolumn{1}{|c|}{ LRT $(3)$} & $\overline{M R C(3)}$ & $\overline{M R T}(3)$ \\
\hline \hline 100 & 0.0771 & 0.0303 & 0.0803 & 0.0231 \\
\hline 150 & 0.0729 & 0.0394 & 0.0754 & 0.0326 \\
\hline 200 & 0.0729 & 0.0477 & 0.0680 & 0.0391 \\
\hline 300 & 0.0606 & 0.0446 & 0.0580 & 0.0420 \\
\hline 500 & 0.0589 & 0.0509 & 0.0571 & 0.0460 \\
\hline \hline
\end{tabular}

From Table 17 is quite evident that the two procedures perform equally well. 
Table 18

10-VARIATE $V A R(2)$, Cointegrated of rank 9

ERF, Trace and Maximal Root Tests

Nominal Size 0.05, normal errors (i.i.d.)

\begin{tabular}{|r||r|r||r|l||c|c||c|c||}
\hline \hline \multicolumn{1}{||c||}{} & \multicolumn{5}{c||}{$\mathrm{T}=75$} & \multicolumn{4}{c||}{$\mathrm{T}=100$} \\
\hline \hline$r_{0}$ & $C R T_{Z}$ & LRT & $M R C$ & MRT & $C R T_{Z}$ & LRT & $M R C$ & MRT \\
\hline \hline 10 & 1.000 & 0.991 & 1.000 & 0.000 & 1.000 & 1.000 & 1.000 & 0.240 \\
\hline 9 & 1.000 & 0.977 & 1.000 & 0.000 & 1.000 & 1.000 & 1.000 & 0.110 \\
\hline 8 & 1.000 & 0.944 & 1.000 & 0.000 & 1.000 & 1.000 & 1.000 & 0.092 \\
\hline 7 & 1.000 & 0.878 & 1.000 & 0.000 & 1.000 & 1.000 & 1.000 & 0.106 \\
\hline 6 & 0.999 & 0.804 & 0.998 & 0.001 & 1.000 & 0.999 & 0.999 & 0.144 \\
\hline 5 & 0.737 & 0.674 & 0.836 & 0.002 & 0.956 & 0.996 & 0.954 & 0.180 \\
\hline 4 & 0.063 & 0.510 & 0.244 & 0.009 & 0.278 & 0.979 & 0.471 & 0.280 \\
\hline 3 & 0.000 & 0.312 & 0.006 & 0.027 & 0.002 & 0.864 & 0.033 & 0.349 \\
\hline 2 & 0.000 & 0.131 & 0.000 & 0.092 & 0.000 & 0.481 & 0.000 & 0.507 \\
\hline 1 & 0.000 & 0.031 & 0.000 & 0.031 & 0.000 & 0.040 & 0.000 & 0.040 \\
\hline \hline
\end{tabular}

Table 19

10-VARIATE $V A R(2)$, Cointegrated of rank 8

ERF, Trace and Maximal Root Tests

Nominal Size 0.05, normal errors (i.i.d.)

\begin{tabular}{||r||r|c||c|c||c|c||c|c||}
\hline \hline \multicolumn{1}{||c||}{} & \multicolumn{5}{c||}{$\mathrm{T}=75$} & \multicolumn{4}{c||}{$\mathrm{T}=100$} \\
\hline \hline$r_{0}$ & $C R T_{Z}$ & LRT & $M R C$ & MRT & $C R T_{Z}$ & LRT & $M R C$ & MRT \\
\hline \hline 10 & 1.000 & 0.984 & 1.000 & 0.000 & 1.000 & 1.000 & 1.000 & 0.334 \\
\hline 9 & 1.000 & 0.936 & 1.000 & 0.000 & 1.000 & 1.000 & 1.000 & 0.154 \\
\hline 8 & 1.000 & 0.856 & 1.000 & 0.000 & 1.000 & 1.000 & 1.000 & 0.097 \\
\hline 7 & 1.000 & 0.729 & 1.000 & 0.000 & 1.000 & 0.998 & 1.000 & 0.091 \\
\hline 6 & 0.998 & 0.583 & 0.998 & 0.000 & 1.000 & 0.990 & 1.000 & 0.104 \\
\hline 5 & 0.711 & 0.386 & 0.868 & 0.003 & 0.917 & 0.933 & 0.966 & 0.142 \\
\hline 4 & 0.049 & 0.206 & 0.311 & 0.006 & 0.147 & 0.711 & 0.567 & 0.196 \\
\hline 3 & 0.000 & 0.065 & 0.013 & 0.014 & 0.000 & 0.239 & 0.056 & 0.235 \\
\hline 2 & 0.000 & 0.012 & 0.000 & 0.011 & 0.000 & 0.031 & 0.000 & 0.023 \\
\hline 1 & 0.000 & 0.008 & 0.000 & 0.008 & 0.000 & 0.009 & 0.000 & 0.009 \\
\hline \hline
\end{tabular}


The conformity test has a tendency toward positive size distortions, while the likelihood ratio test has a tendency toward negative size distortions. In the case of the latter case one sees the strong influence exerted by the imposition of the (false) null. One might argue that, at least in the case of the likelihood ratio test these (trace) tests are moot since, under the null (that all three roots are zero) the undelying sequence is not cointegrated and, thus, it violates a basic assumption involved in estimation.

\subsection{Performance of the Standard $C C T$ Tests}

In Tables $20 \mathrm{~A}$ and $20 \mathrm{~B}$ we give the empirical rejection frequencies for the "standard" conformity tests; these tests involve hypotheses regarding the sum of roots, at least one of which is nonnull. In such cases we have a well developed asymptotic theory, and the results presented therein indicate considerable power, both for the trivariate and the quadrivariate design. Notice that here we actually test hypotheses that the sum is not null by means of confidence intervals. For simplicity of comparison with the earlier results we report our findings as if the hypotheses asserted that the sum of the roots in question were null, when in fact we know, from the design that they are not. Thus, although the tables read as if they report on the rejection of a false null, in fact they report on the acceptance of a true null.

Table 20A

TRIVARIATE $V A R(3)$

Empirical Rejection Frequencies for CCT and CRT Tests.

\begin{tabular}{||r|r|r|r|r|r|r||}
\hline \hline \multicolumn{1}{|c|}{ Test } & PC1 & PC2 & \multicolumn{1}{|c||}{ PC3 } & PC4 & PC5 & PC6 \\
\hline \hline \multicolumn{7}{|c||}{ T $=100$} \\
\hline \hline CCT & 1.00 & 1.00 & 0.9993 & 0.9993 & 1.00 & 0.9996 \\
\hline CRT(2) & 1.00 & N.A. & 1.00 & N.A. & 0.9923 & N.A. \\
\hline CRT(1) & N.A. & N.A. & N.A. & N.A. & N.A. & N.A. \\
\hline \hline \multicolumn{7}{|c||}{ T $=500$} \\
\hline \hline CCT & 1.00 & 1.00 & 1.00 & 1.00 & 1.00 & 1.00 \\
\hline CRT(2) & 1.00 & N.A. & 1.00 & N.A. & 1.00 & N.A. \\
\hline CRT(1) & N.A. & N.A. & N.A. & N.A. & N.A. & N.A. \\
\hline \hline
\end{tabular}


Table 20B

QUADRIVARIATE $V A R(4)$

Empirical Rejection Frequencies for CCT and CRT Tests.

\begin{tabular}{||r|r|r|r|r|r|r|r|r||||}
\hline \hline Test & PC1 & PC2 & PC3 & \multicolumn{1}{|c||}{ PC4 } & PC5 & PC6 & PC7 & PC8 \\
\hline \hline \multicolumn{8}{|c||}{ T $=100$} \\
\hline \hline CCT & 1.00 & 1.00 & 1.00 & 0.9997 & 0.9997 & 1.00 & 1.00 & 1.00 \\
\hline CRT(3) & 1.00 & 1.00 & N.A. & 1.00 & 0.9993 & 1.00 & 0.9993 & N.A. \\
\hline CRT(2) & 1.00 & N.A. & N.A. & 0.9983 & N.A. & 1.00 & N.A. & N.A. \\
\hline CRT(1) & N.A. & N.A. & N.A. & N.A. & N.A. & N.A. & N.A. & N.A. \\
\hline \hline \multicolumn{8}{|c|}{ T $=500$} \\
\hline \hline CCT & 1.00 & 1.00 & 1.00 & 1.00 & 1.00 & 1.00 & 1.00 & 1.00 \\
\hline CRT(3) & 1.00 & 1.00 & N.A. & 1.00 & 1.00 & 1.00 & 1.00 & N.A. \\
\hline CRT(2) & 1.00 & N.A. & N.A. & 1.00 & N.A. & 1.00 & N.A. & N.A. \\
\hline CRT(1) & N.A. & N.A. & N.A. & N.A. & N.A. & N.A. & N.A. & N.A. \\
\hline \hline
\end{tabular}

\section{Conclusions}

The present study explores the properties of three system-based cointegration tests: the newly proposed Conformity Test for Cointegration, Dhrymes (1996b), the likelihood ratio test, Johansen (1988), (1991), and Stock and Watson's $q_{f}(k, m)$ test, Stock and Watson (1988). The main contribution of the paper is a detailed study of the finite sample properties of these tests.

In carrying out this study we have employed a new method for the design of the data generating process, (DGP), here a cointegrated VAR(n), that allows us to specify the characterisitc roots of the system at will. Computational problems confined our full scale analysis, at most, to a quadrivariate VAR(4) with one, two and three unit roots, and stationary roots which were alternatively small, medium, and large (up to .95); there was also a limited examinatin of trivariate random walks and 10 -variate systems with one and two unit roots only. The procedure for obtaining such parametric specifications is outlined in Dhrymes (1996a).

The DGP we have actually employed were two VARs with coefficient matrices which are unconstrained beyond the specification of the system's roots; in particular we have employed VARs with three variables and three lags, and four variables and four lags, and we have used two sample sizes, 100 and 500. The first sample size is used widely in other Monte Carlo studies of this sort, 
and is also found frequently in empirical work. The other sample size has not been used before. In a recent study, Toda (1995) uses a sample of 400. The presumption is that for $\mathrm{T}=500$ asymptotics theory offers a very satisfactory approximation to the actual distribution of the estimators and the test statistics.

The baseline error process used was zero mean i.i.d. normal errors, as specified in the standard VAR. We have also considered two additional error processes: moving average errors of order 3 and 4, and centered chi-square errors (with one degree of freedom).

Our findings, concerning the performance of the tests, can be briewfly stated as follows: First, for the case of i.i.d. normal errors, the $C R T_{Z}$ and $L R T$ tests exhibit quite good characteristics, even for sample size $\mathrm{T}=100$, ut the $C R T_{Z}$ tests suffers relatively more from size distortions, while the $L R T$ has severe power problems.

The $C R T_{Z}$ test was found to have size distortions, occasionally of relatively high magnitude, only in $\mathrm{PC}$ with large stationary roots, and more than one unit roots. For the smaller sample, $\mathrm{T}=100$, the conformity test has by far better power than the likelihood ratio test. While the latter lacks power in situations where the stationary roots of the VAR are small, the power performance of the conformity test is affected only when the $\mathrm{PC}$ in question has large stationary roots. In such 'difficult' parametric configurations we have found that the maximal root version of the cointegration test has, almost always higher power and, on occasion, substantially higher power; accordingly, we recommend it as a supplement to the trace $\left(C R T_{Z}\right)$ test. The maximal root version of the likelihood ratio test $(M R T)$ does not exhibit substantial differences relative to the trace ( $L R T$ ) test. For the larger sample, $\mathrm{T}=500$, the power performance of the two tests is almost identical.

The performance of the $q_{f}(k, m)$ test is very weak and inconclusive, a result also obtained by Yap and Reinsel (1995). While in some instances the test exhibits high power in detecting cointegration, i.e. rejecting that the smallest root is unity, it also frequently rejects that the largest root is unity, thus concluding that the series in question is stationary.

As the dimension of the system under study increases, from $q=3$ to $q$ $=4$, we have observed, for $\mathrm{T}=100$, power losses for both the $C R T_{Z}$ and $L R T$ tests, even in PC with small stationary roots. While the power differences between the two tests in the smaller system were not large, in the larger (quadrivariate) system the $L R T$ test is considerably less powerful, by as much as $30 \%$ in some $\mathrm{PCs}$, in comparison to the $C R T_{Z}$ test. The general result that emerges from this study is that, potentially, both tests can underestimate the true cointegrating rank but the $L R T$ test is much more likely to do so than the $C R T_{Z}$ test. 
When moving average errors were used as the error process in the DGP we have observed increased power at some instances; this may be attributable to the overestimation of the non-zero roots in both the $C R T_{Z}$ and $L R T$. But the size distortions are now much more severe for both tests. An important and interesting finding in this case is that as the sample size increases the size distortions for the LRT test increase rather than decrease, se for example Tables 13A and 13B, thus making the use of the LRT test especially susceptible to error.

On the other hand, when non-symmetric $\chi^{2}$ errors are used we have observed no size problems, as in the case of i.i.d. normal errors. Loss of power was observed but it was limited to the case of 'difficult' PC, with large stationary roots and in general disappear when a sample of $\mathrm{T}=500$ was used.

The two finding regarding alternative error processes are easily understood if we note that with $\mathrm{MA}(3)$ or MA(4) errors the estimation procedure employed renders the asymptotic theory on which the tests are based invalid. Thus, the empirical performance of the tests need not improve with an increase in sample size. On the other hand, the asymptotic theory on which the tests are based remains valid when the error process is i.i.d. with distribution $\chi_{1}^{2}-1$.

The conformity test based on the nonzero roots, which has a standard limiting distribution, is extremely effective in establishing the presence of cointegration, but is not easily employed in establishing the rank of cointegration. This is due to the fact that it lacks power against alternatives that involve sums of zero roots.

In view of the above we conclude that much better inference is to be obtained by the simultaneous use of the $C R T_{Z}$ and the $L R T$ tests. Their design differences should allow a researcher to better capture the probabilistic properties of the series under study. Once cointegration has been established, which both tests achieve without any difficulty, the search for the cointegrating rank should be done using both procedures; $L R T$ is likely to underestimate the rank, as Toda (1995) also reports, due to power problems, and the $C R T_{Z}$ test can readily help in reducing this potential. Finally, based on our results, and in agreement with Yap and Reinsel (1995), we cannot recommend the $q_{f}(k, m)$ test for empirical use.

One last comment on the behavior of the the likelihood ratio test. This test is effectively based on statistics which reflect the cointegration hypothesis, i.e. the residual matrices $V$ and $W$ employed in such test reflect the rank factorization $B \Gamma^{\prime}$ imposed by the null. When the null is true this leads to a net benefit for $L R T$, but when it is false, it seems to result in apreciable loss of power. This seems to be what comes through a great deal of the Monte Carlo results presented earlier. In this light, and whith relatively small samples, the use of the Conformity test in empirical research seems to be highly desirable. 
Our work can be extended to several directions. A major field for extensions would be misspesification analysis: Using the design adopted by this study, we can test for the effects of lag misspecification in cointegrated $V A R(n)$ models, with $n$ arbitrary. According to the results of Boswijk and Frances (1992), for the LRT test, underspecification leads to size distortions whereas overspecification leads to power loss; one can examine this issue in the context of the conformity test.

Given our results on the models with moving average errors, one can extend the results of Van Giersbergen (1996) on the use of bootstrapping, which can be applied to the conformity framework. Finally, one can consider the effects of the presence or absence of trends in the data and estimation and examine the impact of such circumstances on the performance of the various tests examined herein. 


\section{REFERENCES}

Ahn, S.K. and G.C. Reinsel (1990), "Estimation for partially non-stationary multivariate autoregressive models", Journal of the American Statistical Association, vol. 85 , pp. 813-823.

Andrews, D.W.K. (1991), "Heteroscedasticity and autocorrelation consistent covariance matrix estimation", Econometrica, vol. 59, pp. 817-858.

Banerjee, A., J.J. Dolado, D.F. Hendry and G.W. Smith (1986), "Exploring equilibrium relationships in econometrics through static models: Some Monte Carlo evidence", Oxford Bulletin of Economics and Statistics, vol. 48, pp. 253277.

Blangiewicz, M. and W. Charemza (1990), "Cointegation in small samples; empirical percentiles, drifting moments and customized testing", Oxford Bulletin of Economics and Statistics, vol. 52, pp. 303-315.

Bewley R. and M. Yang (1995), "Tests for Cointegration Based on Canonical Correlation Analysis", Journal of the American Statistical Association, vol. 90, pp. 990-996.

Boswijk, H.P. (1994), "Testing for an Unstable Root in Conditional and Structural Error Correction Models", Journal of Econometrics, vol. 63, pp. 37-60.

Boswijk, H.P. and Ph.H. Frances (1992), "Dynamic Specification and Cointegration", Oxford Bulletin of Economics and Statistics, vol. 55, pp. 313-328.

Cheung, Y. and K.S. Lai (1993), "Finite Sample Sizes of Johansen's Likelihood Ratio Tests for Cointegration", Oxford Bulletin of Economics and Statistics, vol. 55, pp. 313-328.

Dhrymes, P.J. (1984), it Mathematics for Econometrics, second edition, New York: Springer-Verlag.

Dhrymes, P.J. (1989), Topics in Advanced Econometrics: vol. I, Probability Foundations, New York: Springer-Verlag.

Dhrymes, P.J. (1996a), "The Design of Monte Carlo Experiments in VAR Systems", Department of Economics, Columbia University Discussion Paper No. 9596-14.

Dhrymes, P.J. (1996b), "A Conformity Test for Cointegration", Department of Economics, Columbia University Discussion Paper, No. 9696-04.

Dhrymes, P.J. (1998), Time Series, Unit Roots and Cointegration, New York: Academic Press. 
Dickey, D.A. and W.A. Fuller (1979), "Distribution of the Estimators for Autoregressive Time Series with a Unit Root", Journal of the American Statistical Association, vol. 74, pp. 420-431.

Dickey, D.A. and W.A. Fuller (1981), "Likelihood Ratio Test Statistics for Autoregressive Time Series with a Unit Root", Econometrica, vol. 49, pp. 10571072 .

Engle, R.F. and C.W.J. Granger (1987), "Cointegration and Error Correction: Representation, Estimation and Testing", Econometrica, vol. 55, pp. 251-276.

Giersbergen van, N.P.A. (1996), "Bootstraping the Trace Statistic in VAR models: Monte Carlo Results and Applications", Oxford Bulletin of Economics and Statistics, vol. 58, pp. 391-408.

Haug, A. (1993), "Residual Based Tests for Cointegration: A Monte Carlo Study of Size Distortions", Economics Letters, vol. 41, pp. 345-351.

Haug, A. (1996), "Cointegration Tests, a Monte Carlo Comparison", Journal of Econometrics, vol. 71, pp. 89-115.

Hooker, M.A. (1993), "Testing for Cointegration: Power versus Frequency of Observation", Economics Letters, vol. 41, pp. 359-362.

Johansen, S. (1988), "Statistical Analysis of Cointegrating Vectors", Journal of Economic Dynamics and Control, vol. 12, pp. 231-254.

Johansen, S. (1991), "Estimation and Hypothesis Testing of Cointegration Vectors in Gaussian Vector Autoregressive Models", Econometrica, vol. 59, pp. 1551-1580.

Johansen, S. and K. Juselius (1990), "Maximum Likelihood Estimation and Inference on Cointegration with an Application to the Demand for Money", Oxford Bulletin of Economics and Statistics, vol. 52, pp. 169-210.

Kleinbergen, F. and K. van Dijik (1994), "Direct Cointegration Testing in Error Correction Models", Journal of Econometrics, vol. 63, pp. 61-103.

Kremers, J.J.M., N.R. Ericsson, and J.J. Dolado (1992), "The Power of Cointegration Tests", Oxford Bulletin of Economics and Statistics, vol. 54, pp. 159-178.

Osterwald-Lenum, M. (1992), "A Note with Quantiles of the Asymptotic Distribution of the Maximum Likelihood Cointegration Rank Test Statistics", Oxford Bulletin of Economics and Statistics, vol. 54, pp. 461-471.

Phillips, P.C.B. (1991), "Optimal Inference In Cointegrated Systems", Econo- 
metrica, vol. 59, pp. 283-306.

Phillips, P.C.B. and S. Ouliaris (1988), "Testing for Cointegration Using Principal Components Methods", Journal of Economic Dynamics and Control, vol. 12, pp. 205-230.

Phillips, P.C.B and S. Ouliaris (1990), "Asymptotic Properties of Residual Based Tests for Cointegration", Econometrica, vol. 58, pp. 165-193.

Reinsel, G.C. and S.K. Ahn (1992), "Vector Autoregressive Models with Unit Roots and Reduced Rank Structure: Estimation, Likelihood Ratio Tests and Forecasting", Journal of Time Series Analysis, vol. 13, pp. 355-375.

Saikkonen, P. (1992), "Estimation and Testing of Cointegrated Systems by an Autoregressive Approximation", Econometric Theory, vol. 8, pp. 1-27.

Shin, Y. (1994), "A Residual Based Test of the Null of Cointegration against the Alternative of no Cointegration", Econometric Theory, vol. 10, pp. 91-115.

Stock, J. and M. Watson (1988), "Testing for Common Trends", Journal of the American Statistical Association, vol. 83, pp. 1097-1107.

Toda, H. (1995), "Finite Sample Performance of Likelihood Ratio Tests for Cointegrating Ranks in Vector Autoregressions", Econometric Theory, vol. 11 no. 5 pp. 1015-1032.

Yap, S.F. and G.C. Reinsel (1995), "Estimation and Testing for Unit Roots in a Partially Non-Stationary Vector Autoregressive Moving Average Model", Journal of the American Statistical Association, vol. 90, pp. 253-267. 


\section{APPENDIX}

In this appendix we present in tabular form a number of results from our study which we did not include in the main body of the paper and on which we did not offer sustained comment.

Table A1

TRIVARIATE $V A R(3)$

$\mathrm{ERF}, M R C\left(r_{0}\right), M R T\left(r_{0}\right)$

$\mathrm{T}=100$, Nominal Size 0.05, normal errors (i.i.d.)

\begin{tabular}{||r|r|r||r|r||r|r||}
\hline \hline Test & $M R C(3)$ & MRT(3) & $\overline{M R C(2)}$ & MRT(2) & $M R C(1)$ & MRT(1) \\
\hline PC1 & 1.00 & 0.99 & 0.99 & 0.93 & 0.01 & 0.05 \\
\hline PC2 & 1.00 & 0.99 & 0.11 & 0.05 & 0.00 & 0.01 \\
\hline PC3 & 1.00 & 0.85 & 0.95 & 0.67 & 0.01 & 0.06 \\
\hline PC4 & 1.00 & 0.55 & 0.37 & 0.06 & 0.00 & 0.01 \\
\hline PC5 & 1.00 & 0.95 & 0.40 & 0.17 & 0.00 & 0.04 \\
\hline PC6 & 1.00 & 0.99 & 0.87 & 0.12 & 0.01 & 0.02 \\
\hline \hline
\end{tabular}

Table A2

TRIVARIATE $V A R(3)$

$\mathrm{ERF}, M R C\left(r_{0}\right), M R T\left(r_{0}\right)$

$\mathrm{T}=500$, Nominal Size 0.05, normal errors (i.i.d.)

\begin{tabular}{||r|r|r||r|r||r|r||}
\hline \hline Test & $M R C(3)$ & MRT(3) & $M R C(2)$ & MRT(2) & $M R C(1)$ & MRT(1) \\
\hline PC1 & 1.00 & 1.00 & 1.00 & 1.00 & 0.00 & 0.05 \\
\hline PC2 & 1.00 & 1.00 & 0.02 & 0.05 & 0.01 & 0.01 \\
\hline PC3 & 1.00 & 1.00 & 1.00 & 1.00 & 0.00 & 0.06 \\
\hline PC4 & 1.00 & 1.00 & 0.07 & 0.06 & 0.00 & 0.01 \\
\hline PC5 & 1.00 & 1.00 & 0.98 & 0.98 & 0.02 & 0.06 \\
\hline PC6 & 1.00 & 1.00 & 0.49 & 0.07 & 0.00 & 0.01 \\
\hline \hline
\end{tabular}


Table A3

TRIVARIATE $V A R(3)$

$\mathrm{ERF}, M R C\left(r_{0}\right), M R T\left(r_{0}\right)$

$\mathrm{T}=100$, Nominal Size 0.05, MA(3) Errors

\begin{tabular}{||r|r|r||r|r||r|r||}
\hline \hline Test & $M R C(3)$ & MRT(3) & $M R C(2)$ & MRT(2) & $M R C(1)$ & MRT(1) \\
\hline PC1 & 1.00 & 1.00 & 1.00 & 1.00 & 0.01 & 0.07 \\
\hline PC2 & 1.00 & 1.00 & 0.57 & 0.32 & 0.00 & 0.02 \\
\hline PC5 & 1.00 & 1.00 & 0.21 & 0.10 & 0.04 & 0.07 \\
\hline PC6 & 1.00 & 1.00 & 0.87 & 0.37 & 0.04 & 0.02 \\
\hline \hline
\end{tabular}

Table A4

TRIVARIATE $V A R(3)$

ERF, $M R C\left(r_{0}\right), M R T\left(r_{0}\right)$

$\mathrm{T}=500$, Nominal Size 0.05, MA(3) Errors

\begin{tabular}{||r|r|r||r|r||r|r||}
\hline \hline Test & $M R C(3)$ & MRT(3) & $M R C(2)$ & MRT(2) & $M R C(1)$ & MRT(1) \\
\hline PC1 & 1.00 & 1.00 & 1.00 & 1.00 & 0.01 & 0.09 \\
\hline PC2 & 1.00 & 1.00 & 0.54 & 0.53 & 0.03 & 0.05 \\
\hline PC5 & 1.00 & 1.00 & 0.95 & 0.98 & 0.03 & 0.17 \\
\hline PC6 & 1.00 & 1.00 & 0.77 & 0.41 & 0.00 & 0.02 \\
\hline \hline
\end{tabular}

Table A5

TRIVARIATE $V A R(3)$

$\mathrm{ERF}, M R C\left(r_{0}\right), M R T\left(r_{0}\right)$

$\mathrm{T}=100$, Nominal Size 0.05, $\chi^{2}$ Errors

\begin{tabular}{||r|r|r||r|r||r|r||}
\hline \hline Test & $M R C(3)$ & MRT(3) & $M R C(2)$ & MRT(2) & $M R C(1)$ & MRT(1) \\
\hline PC1 & 1.00 & 0.99 & 0.98 & 0.89 & 0.00 & 0.05 \\
\hline PC2 & 1.00 & 0.96 & 0.08 & 0.04 & 0.00 & 0.01 \\
\hline PC5 & 1.00 & 0.89 & 0.21 & 0.08 & 0.00 & 0.02 \\
\hline PC6 & 0.99 & 0.47 & 0.71 & 0.06 & 0.00 & 0.02 \\
\hline \hline
\end{tabular}


Table A6

TRIVARIATE $V A R(3)$

$\mathrm{ERF}, M R C\left(r_{0}\right), M R T\left(r_{0}\right)$

$\mathrm{T}=500$, Nominal Size $0.05, c h i^{2}$ Errors

\begin{tabular}{||r|r|r||r|r||r|r||}
\hline \hline Test & $M R C(3)$ & MRT(3) & $M R C(2)$ & MRT(2) & $M R C(1)$ & MRT(1) \\
\hline PC1 & 1.00 & 1.00 & 1.00 & 1.00 & 0.00 & 0.05 \\
\hline PC2 & 1.00 & 1.00 & 0.02 & 0.04 & 0.00 & 0.00 \\
\hline PC5 & 1.00 & 1.00 & 0.52 & 0.62 & 0.00 & 0.05 \\
\hline PC6 & 1.00 & 1.00 & 0.59 & 0.06 & 0.00 & 0.00 \\
\hline \hline
\end{tabular}

Table A7

QUADRIVARIATE $V A R(4)$

$\mathrm{ERF}, M R C\left(r_{0}\right), M R T\left(r_{0}\right), \mathrm{T}=100$

Nominal Size 0.05, normal errors (i.i.d.)

\begin{tabular}{||r|r|r||r|r||}
\hline \hline Test & $\overline{M R C(4)}$ & MRT(4) & $\bar{M} R \overline{(3)}$ & MRT(3) \\
\hline PC1 & 1.00 & 1.00 & 0.99 & 0.70 \\
\hline PC2 & 1.00 & 1.00 & 0.97 & 0.59 \\
\hline PC3 & 1.00 & 1.00 & 0.58 & 0.09 \\
\hline PC4 & 1.00 & 1.00 & 0.99 & 0.28 \\
\hline PC5 & 1.00 & 1.00 & 0.86 & 0.21 \\
\hline PC6 & 1.00 & 0.90 & 0.98 & 0.33 \\
\hline PC7 & 1.00 & 0.99 & 0.87 & 0.18 \\
\hline PC8 & 1.00 & 0.99 & 0.90 & 0.20 \\
\hline & & & & \\
\hline \hline Test & $M R C(2)$ & MRT(2) & $M \overline{R C}(1)$ & MRT(1) \\
\hline \hline PC1 & 0.87 & 0.56 & 0.00 & 0.04 \\
\hline PC2 & 0.09 & 0.05 & 0.00 & 0.01 \\
\hline PC3 & 0.03 & 0.01 & 0.00 & 0.00 \\
\hline PC4 & 0.27 & 0.06 & 0.00 & 0.01 \\
\hline PC5 & 0.16 & 0.05 & 0.00 & 0.02 \\
\hline PC6 & 0.35 & 0.12 & 0.00 & 0.04 \\
\hline PC7 & 0.15 & 0.03 & 0.00 & 0.01 \\
\hline PC8 & 0.21 & 0.04 & 0.00 & 0.02 \\
\hline \hline
\end{tabular}


Table A8

QUADRIVARIATE $V A R(4)$

ERF, $M R C\left(r_{0}\right), M R T\left(r_{0}\right), \mathrm{T}=500$

Nominal Size 0.05, normal errors (i.i.d.)

\begin{tabular}{||r|r|r||r|r||}
\hline \hline Test & $M R C(4)$ & MRT(4) & $M R C(3)$ & MRT(3) \\
\hline PC1 & 1.00 & 1.00 & 1.00 & 1.00 \\
\hline PC2 & 1.00 & 1.00 & 1.00 & 1.00 \\
\hline PC3 & 1.00 & 1.00 & 0.08 & 0.05 \\
\hline PC4 & 1.00 & 1.00 & 1.00 & 1.00 \\
\hline PC5 & 1.00 & 1.00 & 0.96 & 0.92 \\
\hline PC6 & 1.00 & 1.00 & 1.00 & 1.00 \\
\hline PC7 & 1.00 & 1.00 & 1.00 & 0.98 \\
\hline PC8 & 1.00 & 1.00 & 0.15 & 0.07 \\
\hline & & & & \\
\hline \hline Test & $M R C(2)$ & MRT(2) & $M R C(1)$ & MRT $(1)$ \\
\hline \hline PC1 & 1.00 & 1.00 & 0.00 & 0.05 \\
\hline PC2 & 0.10 & 0.05 & 0.00 & 0.00 \\
\hline PC3 & 0.00 & 0.00 & 0.00 & 0.00 \\
\hline PC4 & 0.51 & 0.75 & 0.00 & 0.06 \\
\hline PC5 & 0.02 & 0.06 & 0.00 & 0.07 \\
\hline PC6 & 1.00 & 1.00 & 0.00 & 0.05 \\
\hline PC7 & 0.02 & 0.04 & 0.00 & 0.01 \\
\hline PC8 & 0.00 & 0.00 & 0.00 & 0.00 \\
\hline \hline
\end{tabular}


Table A9

QUADRIVARIATE $V A R(4)$

$\mathrm{ERF}, M R C\left(r_{0}\right), M R T\left(r_{0}\right), \mathrm{T}=100$

Nominal Size 0.05, MA(4) Errors

\begin{tabular}{||r|r|r||r|r||}
\hline \hline Test & $M R C(4)$ & MRT(4) & $M R C(3)$ & MRT(3) \\
\hline PC1 & 1.00 & 1.00 & 1.00 & 1.00 \\
\hline PC2 & 1.00 & 1.00 & 1.00 & 1.00 \\
\hline PC4 & 1.00 & 1.00 & 1.00 & 1.00 \\
\hline PC5 & 1.00 & 1.00 & 1.00 & 0.62 \\
\hline & & & & \\
\hline \hline Test & $M R C(2)$ & MRT(2) & $M R C(1)$ & MRT(1) \\
\hline \hline PC1 & 1.00 & 1.00 & 0.00 & 0.05 \\
\hline PC2 & 0.82 & 0.05 & 0.00 & 0.00 \\
\hline PC4 & 1.00 & 1.00 & 0.13 & 0.16 \\
\hline PC5 & 0.82 & 0.15 & 0.05 & 0.03 \\
\hline
\end{tabular}

Table A10

QUADRIVARIATE $V A R(4)$

ERF

$M R C\left(r_{0}\right), M R T\left(r_{0}\right), \mathrm{T}=500$

Nominal Size 0.05, MA(4) Errors

\begin{tabular}{||r|r|r||r|r||}
\hline \hline Test & $M R C(4)$ & MRT(4) & $M R C(3)$ & MRT(3) \\
\hline PC1 & 1.00 & 1.00 & 1.00 & 1.00 \\
\hline PC2 & 1.00 & 1.00 & 1.00 & 1.00 \\
\hline PC4 & 1.00 & 1.00 & 1.00 & 1.00 \\
\hline PC5 & 1.00 & 1.00 & 1.00 & 1.00 \\
\hline & & & & \\
\hline \hline Test & $M R C(2)$ & MRT(2) & $M R C(1)$ & MRT(1) \\
\hline \hline PC1 & 1.00 & 1.00 & 0.00 & 0.05 \\
\hline PC2 & 0.64 & 0.05 & 0.00 & 0.00 \\
\hline PC4 & 1.00 & 1.00 & 0.00 & 0.10 \\
\hline PC5 & 0.54 & 0.19 & 0.00 & 0.01 \\
\hline
\end{tabular}


Table A11

QUADRIVARIATE $V A R(4)$

ERF, $M R C\left(r_{0}\right), M R T\left(r_{0}\right), \mathrm{T}=100$

Nominal Size $0.05, \chi^{2}$ Errors

\begin{tabular}{||r|r|r||r|r||}
\hline \hline Test & \multicolumn{1}{|c|}{$M R C(4)$} & MRT(4) & $M R C(3)$ & MRT(3) \\
\hline PC1 & 1.00 & 0.97 & 1.00 & 0.68 \\
\hline PC2 & 1.00 & 1.00 & 1.00 & 0.74 \\
\hline PC4 & 1.00 & 0.94 & 1.00 & 0.30 \\
\hline PC5 & 1.00 & 0.95 & 0.78 & 0.16 \\
\hline & & & & \\
\hline \hline Test & $M R C(2)$ & MRT(2) & $M R C(1)$ & MRT(1) \\
\hline \hline PC1 & 0.81 & 0.52 & 0.00 & 0.04 \\
\hline PC2 & 0.08 & 0.05 & 0.00 & 0.01 \\
\hline PC4 & 0.24 & 0.06 & 0.01 & 0.03 \\
\hline PC5 & 0.11 & 0.04 & 0.00 & 0.02 \\
\hline
\end{tabular}

Table A12

QUADRIVARIATE $V A R(4)$

$\mathrm{ERF}, M R C\left(r_{0}\right), M R T\left(r_{0}\right), \mathrm{T}=500$

Nominal Size 0.05, $\chi^{2}$ Errors

\begin{tabular}{||r|r|r||r|r||}
\hline \hline Test & $M R C(4)$ & MRT(4) & $M R C(3)$ & MRT(3) \\
\hline PC1 & 1.00 & 1.00 & 1.00 & 1.00 \\
\hline PC2 & 1.00 & 1.00 & 1.00 & 1.00 \\
\hline PC4 & 1.00 & 1.00 & 1.00 & 1.00 \\
\hline PC5 & 1.00 & 1.00 & 0.90 & 0.85 \\
\hline & & & & \\
\hline \hline Test & $M R C(2)$ & MRT(2) & $M R C(1)$ & MRT(1) \\
\hline \hline PC1 & 1.00 & 1.00 & 0.00 & 0.05 \\
\hline PC2 & 0.00 & 0.05 & 0.00 & 0.01 \\
\hline PC4 & 0.38 & 0.72 & 0.00 & 0.05 \\
\hline PC5 & 0.01 & 0.06 & 0.00 & 0.02 \\
\hline
\end{tabular}




\title{
1997-1998 Discussion Paper Series
}

\author{
Department of Economics \\ Columbia University \\ 1022 International Affairs Bldg. \\ 420 West 118th Street \\ New York, N.Y., 10027
}

The following papers are published in the 1997-98 Columbia University Discussion Paper series which runs from early November to October 31 of the following year (Academic Year).

The following is a website address where viewers may download some discussion papers. It is a project that is in progress, and therefore the site may not contain all recent working papers.

http://www.columbia.edu/dlc/wp/econ/index.html.

For Ordering Hardcopies:

Domestic orders for discussion papers are available for purchase at the cost of $\$ 8.00$ (U.S.) Per paper and $\$ 140.00$ (US) for the series.

Foreign orders cost $\$ 10.00$ (US) per paper and $\$ 185.00$ for the series.

To order discussion papers, please write to the Discussion Paper Coordinator at the above address along with a check for the appropriate amount, made payable to Department of Economics, Columbia University. Please be sure to include the series number of the requested paper when you place an order. 
1997-98 Discussion Papers

Economics Department, Columbia University

\begin{tabular}{|c|c|c|}
\hline Seriesno & 5. Titie & 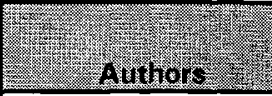 \\
\hline $9798-01$ & Optimal Regulatory Transparency & Herk, L.F. \\
\hline$\overline{9798-02}$ & Russia's Market Transition: Who Has Lost and How? & $\begin{array}{l}\text { Desai, } \mathrm{P} . \\
\text { Idson, } \mathrm{T} \text {. }\end{array}$ \\
\hline $9798-03$ & The Wage Arrears Crisis in Russia & $\begin{array}{l}\text { Desai, } \mathrm{P} \text {. } \\
\text { Idson, } \mathrm{T} \text {. }\end{array}$ \\
\hline $9798-04$ & Taxes Versus Legal Rules as Instruments for Equity & Sanchirico, C.W. \\
\hline $9798-05$ & Enforcement by Hearing: An Integrated Model of Evidence Production & Sanchirico, C.W. \\
\hline $9798-06$ & $\begin{array}{l}\text { Evidence Production, Adversarial Process and the Private Instigation of Suits } \\
12 / 95)\end{array}$ & Sanchirico, C.W. \\
\hline $9798-07$ & Structural VAR, MARMA and Open Economy Models & $\begin{array}{l}\text { Dhrymes, P.J. } \\
\text { Thomakos, D. }\end{array}$ \\
\hline $9798-08$ & $\begin{array}{l}\text { Small Sample Properties of Certain Cointegration Test Statistics: A Monte Carlo Study (Revd. } \\
\text { from 7/96) }\end{array}$ & $\begin{array}{l}\text { Dhrymes, P.J. } \\
\text { Thomakos, D. }\end{array}$ \\
\hline
\end{tabular}

